\title{
INVARIANT SUBSPACES IN BANACH SPACES OF ANALYTIC FUNCTIONS
}

\author{
STEFAN RICHTER
}

\begin{abstract}
We study the invariant subspace structure of the operator of multiplication by $z, M_{z}$, on a class of Banach spaces of analytic functions. For operators on Hilbert spaces our class coincides with the adjoints of the operators in the CowenDouglas class $\mathscr{B}_{1}(\bar{\Omega})$. We say that an invariant subspace $\mathscr{M}$ satisfies $\operatorname{cod} \mathscr{M}=1$ if $z \mathscr{M}$ has codimension one in $\mathscr{M}$. We give various conditions on invariant subspaces which imply that $\operatorname{cod} \mathscr{M}=1$. In particular, we give a necessary and sufficient condition on two invariant subspaces $\mathscr{M}, \mathscr{N}$ with $\operatorname{cod} \mathscr{M}=\operatorname{cod} \mathscr{N}=1$ so that their span again satisfies $\operatorname{cod}(\mathscr{M} \vee \mathscr{N})=1$. This result will be used to show that any invariant subspace of the Bergman space $L_{a}^{p}, p \geqslant 1$, which is generated by functions in $L_{a}^{2 p}$, must satisfy $\operatorname{cod} \mathscr{M}=1$. For an invariant subspace $\mathscr{M}$ we then consider the operator $S=M_{z}^{*} \mid \mathscr{M}^{\perp}$. Under some extra assumption on the domain of holomorphy we show that the spectrum of $S$ coincides with the approximate point spectrum iff $\operatorname{cod} \mathscr{M}=1$. Finally, in the last section we obtain a structure theorem for invariant subspaces with $\operatorname{cod} \mathscr{M}=1$. This theorem applies to Dirichlet-type spaces.
\end{abstract}

\section{Introduction.}

1.1. Overview. Let $\Omega$ be an open and connected subset of the complex plane. The Banach spaces $\mathscr{B}$ under consideration satisfy the following axioms:

$\mathscr{B}$ is a vector subspace of the space of all analytic functions on $\Omega$.

The functional of evaluation at $\lambda$ is continuous for all $\lambda \in \Omega$.

If $f$ is a function in $\mathscr{B}$, then so is $z f$.

If $f \in \mathscr{B}$ and $f(\lambda)=0$, then there is a function $g \in \mathscr{B}$ such that $(z-\lambda) g=f$.

It follows immediately from the first three axioms and the closed graph theorem that the linear transformation $f \mapsto z f$ is continuous. We will denote it by $\left(M_{z}, \mathscr{B}\right)$ or $M_{z}$ if the underlying Banach space has been fixed.

In $\$ 2$ we shall discuss some examples of spaces satisfying these axioms. In particular, Theorem 2.10 states that if $\mathscr{B}$ is a Hilbert space, then the Hilbert space adjoints $M_{z}^{*}$ of $M_{z}$ exactly form the Cowen-Douglas class $\mathscr{B}_{1}(\bar{\Omega})$ (see $\S 2.4$ for a definition).

Received by the editors December 12, 1986.

1980 Mathematics Subject Classification (1985 Revision). Primary 47B38; Secondary 46E15.

This work appeared as part of the author's Ph.D. dissertation at the University of Michigan (1986). The author would like to acknowledge the many helpful conversations he has had with Allen Shields. 
A subspace $\mathscr{M}$ of $\mathscr{B}$ will always be a closed subspace, and it is called invariant if $M_{z}$ maps $\mathscr{M}$ into itself. In [14] the authors suggested to study the operators $M_{z} \mid \mathscr{M}$ in the hope of obtaining some information about the invariant subspace $\mathscr{M}$. In [22] the author showed that for a large class of Hilbert spaces of analytic functions the operators $M_{z} \mid \mathscr{M}$ and $M_{z} \mid \mathscr{N}$ are unitarily equivalent if and only if the invariant subspaces $\mathscr{M}$ and $\mathscr{N}$ coincide.

Our approach in the present article is to consider one of the unitary invariants of the operators $M_{z} \mid \mathscr{M}$ : the Fredholm index. Under our assumptions (1.1)-(1.4) we shall show that for every nonzero invariant subspace $\mathscr{M},-\operatorname{index}\left(\left(M_{z}-\lambda\right) \mid \mathscr{M}\right)=$ the codimension of $(z-\lambda) \mathscr{M}$ in $\mathscr{M}$ does not depend on $\lambda$ in the domain of holomorphy. We shall say that the invariant subspace $\mathscr{M}$ has the codimension $n$ property if $-\operatorname{index}\left(\left(M_{z}-\lambda\right) \mid \mathscr{M}\right)=n$ (see Definition 2.12).

Let $L_{a}^{2}$ be the space of all analytic functions in the unit disc which are square integrable with respect to Lebesgue area measure. It follows from results of Apostol, Bercovici, Foiaş, and Pearcy that if $n$ is a positive integer or $\infty$, then there exists an invariant subspace of $L_{a}^{2}$ which has the codimension $n$ property.

Cyclic invariant subspaces have the codimension one property (Corollary 3.3), thus every invariant subspace is the span of invariant subspaces with the codimension one property. In $\$ 3$ we shall give several sufficient conditions for invariant subspaces to have the codimension one property. In particular, Theorem 3.10 gives necessary and sufficient conditions for the span of two invariant subspaces with the codimension one property to again have the codimension one property.

In $\S 4$ we investigate $\sigma\left(M_{z}^{*} \mid \mathscr{M}^{\perp}\right)$ and $\sigma_{\text {ap }}\left(M_{z}^{*} \mid \mathscr{M}^{\perp}\right)$. From this we shall be able to give a sufficient condition on an invariant subspace $\mathscr{M}$ to have the property that all larger invariant subspaces $\mathscr{N} \supseteq \mathscr{M}$ have the codimension one property (Corollary 4.8). Finally, in $\S 5$ we shall restrict our attention to Hilbert spaces $\mathscr{H}$ of analytic functions on the unit disc. In Theorem 5.3 we shall show that if $\sigma\left(M_{z}\right)=\mathbf{D}^{-}$and $\left\|M_{z} f\right\| \geqslant\|f\|$ for all $f \in \mathscr{H}$, then every invariant subspace $\mathscr{M}$ with the codimension one property satisfies $\mathscr{M} \subseteq H^{2} f_{0} \cap \mathscr{H}$ for $f_{0} \in \mathscr{M} \ominus z \mathscr{M}$.

Throughout this paper we have tried to illustrate our abstract operator theoretic theorems with concrete examples from function theory. This should not obscure the fact that the function theoretic aspect of a characterization of the invariant subspaces of most spaces remains widely open. As an example consider again $L_{a}^{2}$. Deep results of Korenblum, Roberts, and H. Shapiro (see [24]) characterize the invariant subspaces that are generated by bounded analytic functions. We shall see that in this context all invariant subspaces must have the codimension one property. A similar statement can be made about invariant subspaces arising from sequences which are universal interpolating for $L_{a}^{2}$ (see [1] or $\S 4$ for a definition).

1.2. Some notations. Most of the notations are either standard or they are defined in the text as we need them. However, there are a few things which we would like to point out here. We use the letter $\mathscr{X}$ to denote an abstract complex Banach space, while $\mathscr{B}$ or $\mathscr{E}$ refer to Banach spaces which satisfy our axioms (1.1)-(1.4) listed above. $\mathscr{H}$ and $\mathscr{K}$ will always refer to Hilbert spaces. $\mathscr{L}(\mathscr{X})$ denotes the algebra of bounded linear transformations on $\mathscr{X}$. 
By $\mathbf{N}$ we mean the set of positive integers, $\mathbf{N}=\{1,2, \ldots\}$. For a set $\Omega \subseteq \mathbf{C}$, we write $\bar{\Omega}$ for the set of complex conjugates, $\bar{\Omega}=\{\omega \in \mathbf{C}: \bar{\omega} \in \Omega\}$. $\Omega^{-}$denotes the closure of $\Omega$.

In our proofs the symbol $\square$ signals the end of a part of the proof, while $\square$ indicates the end of the whole proof.

\section{Banach spaces of analytic functions.}

2.1. Preliminaries. Now suppose $\Omega$ is an open and connected subset of the complex plane and $\mathscr{B}$ satisfies the axioms (1.1)-(1.4).

If $\lambda \in \Omega$ and $\mathscr{B} \neq\{0\}$, then (1.4) implies that there is a function $f \in \mathscr{B}$ with $f(\lambda) \neq 0$; thus the operator $\left(M_{z}-\lambda\right)$ cannot be surjective. This implies that $\Omega \subseteq \sigma\left(M_{z}\right)$, and we see that there is no loss in generality if we assume $\Omega$ to be bounded.

Note that $\Omega$ is not assumed to be maximal in any sense, i.e. if $\Omega^{\prime} \subseteq \Omega, \Omega^{\prime} \neq \varnothing$, and $\mathscr{B}$ is a Banach space of analytic functions on $\Omega$, then $\mathscr{B}$ can also be considered to be a Banach space of analytic functions on $\Omega^{\prime}$, just by restricting the functions in $\mathscr{B}$ to $\Omega^{\prime}$.

Lemma 2.1. Suppose $\mathscr{B}$ satisfies (1.1)-(1.3) and $\mathscr{B} \neq\{0\}$. Then (1.4) is equivalent to each of the following three sets of conditions.

(i) For every $\lambda \in \Omega$ there is a $g \in \mathscr{B}$ with $g(\lambda) \neq 0$,

(ii) for every $\lambda \in \Omega\left(M_{z}-\lambda\right)$ is bounded below,

(iii) there is a $\lambda_{0} \in \Omega$ such that whenever $f \in \mathscr{B}$ and $f\left(\lambda_{0}\right)=0$, there exists a $g \in \mathscr{B}$ such that $\left(z-\lambda_{0}\right) g=f$.

(i) For every $\lambda \in \Omega$ there is a $g \in \mathscr{B}$ with $g(\lambda) \neq 0$,

(ii) for every $\lambda \in \Omega\left(M_{z}-\lambda\right)$ is bounded below,

(iii) $\operatorname{dim} \operatorname{ker}\left(M_{z}-\lambda_{0}\right)^{*}=1$ for some $\lambda_{0} \in \Omega$.

(i) For every $\lambda \in \Omega$ there is a $g \in \mathscr{B}$ with $g(\lambda) \neq 0$,

(ii) for every $\lambda \in \Omega\left(M_{z}-\lambda\right)$ is bounded below,

(iii) $\operatorname{dim} \operatorname{ker}\left(M_{z}-\lambda\right)^{*}=1$ for every $\lambda \in \Omega$.

Proof. Note that $\left(M_{z}-\lambda\right)$ is an injective operator for all $\lambda \in \Omega$; thus it is bounded below if and only if it has a closed range. In this case $M_{z}-\lambda$ is a semi-Fredholm operator and the Fredholm index equals the dimension of the kernel of the adjoint $\left(M_{z}-\lambda\right)^{*}$. Thus the implication $(2.2) \Rightarrow(2.3)$ follows immediately from the general theory of semi-Fredholm operators (see [17]).

To show that $(2.1) \Rightarrow(2.2)$ we only have to establish that $(2.2)$ (iii) follows from (2.1). We note that (2.1)(iii) says that

$$
\operatorname{ran}\left(M_{z}-\lambda_{0}\right)=\left\{f \in \mathscr{B}: f\left(\lambda_{0}\right)=0\right\} .
$$


But clearly the right side of (2.4) has codimension one in $\mathscr{B}$, hence $\operatorname{dim} \operatorname{ker}\left(M_{z}-\lambda_{0}\right)^{*}=$ codimension of $\operatorname{ran}\left(M_{z}-\lambda_{0}\right)$ in $\mathscr{B}=1$.

Next we shall show that (1.4) implies (2.1). Of course (2.1)(iii) follows trivially from (1.4). We saw earlier that (2.1)(i) is valid, if $\mathscr{B} \neq\{0\}$. To show that (2.1)(ii) is satisfied we note that (1.4) implies that (2.4) is valid for all $\lambda \in \Omega$. That the right side of (2.4) is closed follows from axiom (1.2), thus $M_{z}-\lambda$ has closed range. By what was said above $M_{z}-\lambda$ is bounded below.

Finally we shall show that (2.3) implies (1.4). To this end let $\lambda \in \Omega$ be fixed. We know that $\operatorname{ran}\left(M_{z}-\lambda\right) \subseteq\{f \in \mathscr{B}: f(\lambda)=0\}$, and we have to show that equality holds, i.e. (2.4) with $\lambda$. But it follows from (2.3)(ii) that $\operatorname{ran}\left(M_{z}-\lambda\right)$ is closed, (2.3)(iii) implies that the codimension of $\operatorname{ran}\left(M_{z}-\lambda\right)$ in $\mathscr{B}$ equals one. This together with the fact that the codimension of $\{f \in \mathscr{B}: f(\lambda)=0\}$ in $\mathscr{B}$ is one (this follows from (2.3)(i)) proves that (2.4) holds.

REMARK 2.2. Since point evaluations are zontinuous linear functionals on $\mathscr{B}$ there are elements $k_{\lambda} \in \mathscr{B}^{*}$ such that $f(\lambda)=\left\langle f, k_{\lambda}\right\rangle$ for all $f \in \mathscr{B}$. It is easy to see that $k_{\lambda} \in \operatorname{ker}\left(M_{z}-\lambda\right)^{*}$. It follows from (1.4) that $k_{\lambda} \neq 0$ for all $\lambda \in \Omega$, thus Lemma 2.1 implies that $k_{\lambda}$ spans $\operatorname{ker}\left(M_{z}-\lambda\right)^{*}$.

2.2. Multipliers. The commutant of the operator $\left(M_{z}, \mathscr{B}\right)$ has a convenient description. We shall need the following definition which applies to a more general situation.

DEFinition 2.3. Suppose $\mathscr{B}_{1}$ and $\mathscr{B}_{2}$ are two Banach spaces of analytic functions satisfying (1.1)-(1.4). A complex valued function $\varphi$ in $\Omega$ is called a multiplier from $\mathscr{B}_{1}$ into $\mathscr{B}_{2}$, if $\varphi \mathscr{B}_{1} \subseteq \mathscr{B}_{2}$. The set of all multipliers from $\mathscr{B}_{1}$ into $\mathscr{B}_{2}$ will be denoted by $M\left(\mathscr{B}_{1}, \mathscr{B}_{2}\right)$ or $M\left(\mathscr{B}_{1}\right)$ if $\mathscr{B}_{1}=\mathscr{B}_{2}$.

Using the closed graph theorem, it is easily seen that every $\varphi \in M\left(\mathscr{B}_{1}, \mathscr{B}_{2}\right)$ defines a bounded linear transformation $M_{\varphi}: f \mapsto \varphi f$ from $\mathscr{B}_{1}$ into $\mathscr{B}_{2}$. Furthermore, since for all $f \in \mathscr{B}_{1}$ both $f$ and $\varphi f$ are analytic functions it follows that every multiplier $\varphi$ has to be an analytic function as well.

The argument in the proof of the following proposition is taken from [26], where a similar theorem for Hilbert spaces is proved.

Proposition 2.4. Suppose $\mathscr{B}_{1}$ and $\mathscr{B}_{2}$ are two Banach spaces of analytic functions satisfying (1.1)-(1.4). Write $M_{z}^{i}$ for $\left(M_{z}, \mathscr{B}_{i}\right), i=1,2$. An operator $T \in \mathscr{L}\left(\mathscr{B}_{1}, \mathscr{B}_{2}\right)$ satisfies $T M_{z}^{1}=M_{z}^{2} T$, if and only if there is a function $\varphi \in M\left(\mathscr{B}_{1}, \mathscr{B}_{2}\right)$ such that $T=M_{\varphi}$.

Proof. It is clear that a multiplication operator $M_{\varphi}$ satisfies $M_{\varphi} M_{z}^{1}=M_{z}^{2} M_{\varphi}$.

Assume now that $T \in \mathscr{L}\left(\mathscr{B}_{1}, \mathscr{B}_{2}\right)$ and $T M_{z}^{1}=M_{z}^{2} T$. For $i=1,2$ and $\lambda \in \Omega$ denote by $k_{\lambda}^{i}$ the elements in $\operatorname{ker}\left(M_{z}^{i}-\lambda\right)^{*}$ representing the evaluation of functions in $\mathscr{B}_{i}$ at $\lambda$. It follows from the hypothesis that $M_{z}^{1 *} T^{*}=T^{*} M_{z}^{2 *}$. We see that for every $\lambda \in \Omega T^{*}$ maps $\operatorname{ker}\left(M_{z}^{2}-\lambda\right)^{*}$ into $\operatorname{ker}\left(M_{z}^{1}-\lambda\right)^{*}$. Thus, since $k_{\lambda}^{i}$ spans $\operatorname{ker}\left(M_{z}^{i}-\lambda\right)^{*}$, there must exist a function $\varphi$ on $\Omega$ such that $T^{*} k_{\lambda}^{2}=\varphi(\lambda) k_{\lambda}^{1}$. We claim that $T=M_{\varphi}$. Indeed, for $f \in \mathscr{B}_{1}$ and $\lambda \in \Omega$ we have

$$
\begin{aligned}
(T f)(\lambda) & =\left\langle T f, k_{\lambda}^{2}\right\rangle=\left\langle f, T^{*} k_{\lambda}^{2}\right\rangle=\left\langle f, \varphi(\lambda) k_{\lambda}^{1}\right\rangle \\
& =\varphi(\lambda)\left\langle f, k_{\lambda}^{1}\right\rangle=\varphi(\lambda) f(\lambda) .
\end{aligned}
$$


Corollary 2.5. The commutant of the operator $\left(M_{z}, \mathscr{B}\right)$ is equal to $\left\{M_{\varphi}\right.$ : $\varphi \in M(\mathscr{B})\}$.

Note that $M(\mathscr{B})$ can be made into a Banach space by defining $\|\varphi\|=\left\|M_{\varphi}\right\|_{\mathscr{L}(\mathscr{B})}$. The commutant of $\left(M_{z}, \mathscr{B}\right)$ is certainly norm closed in $\mathscr{L}(\mathscr{B})$, thus $M(\mathscr{B})$ is complete in its norm.

Proposition 2.6. If $\mathscr{B}$ satisfies the axioms (1.1)-(1.4) then

$$
\begin{gathered}
M(\mathscr{B}) \subseteq H^{\infty}(\Omega), \\
|\varphi(\lambda)| \leqslant\left\|M_{\varphi}\right\|_{\mathscr{L}(\mathscr{B})}=\|\varphi\|_{M(\mathscr{B})} \quad \text { for all } \lambda \in \Omega .
\end{gathered}
$$

Furthermore, $M(\mathscr{B})$ satisfies (1.1)-(1.4).

Proof. We shall only prove that $M(\mathscr{B})$ satisfies (1.4). (2.5) and (2.6) are covered by Proposition 3 of [9]. This implies that $M(\mathscr{B})$ satisfies (1.1) and (1.2). That $M(\mathscr{B})$ satisfies (1.3) is trivial.

If $\varphi \in M(\mathscr{B}), \varphi(\lambda)=0$, then there is a holomorphic function $\psi$ such that $(z-\lambda) \psi=\varphi$.

Claim. $\psi \in M(\mathscr{B})$.

If $f \in \mathscr{B}$, then

$$
\psi f=\frac{z-\lambda}{z-\lambda} \psi f=\frac{\varphi f}{z-\lambda} .
$$

But $\varphi f \in \mathscr{B}, \varphi(\lambda) f(\lambda)=0$. Thus $\varphi f /(z-\lambda) \in \mathscr{B}$.

Therefore we have $\psi f \in \mathscr{B}$ for every $f \in \mathscr{B}$, hence $\psi \in M(\mathscr{B})$.

At this point note that $M(\mathscr{B})$ always contains the polynomials. On the other hand, if also $1 \in \mathscr{B}$, then $M(\mathscr{B}) \subseteq \mathscr{B} \cap H^{\infty}(\Omega)$.

2.3. Examples. In the following examples $\Omega$ will denote a bounded region in the complex plane.

EXAMPLE 2.7. The Banach algebra $H^{\infty}(\Omega)$ of all bounded analytic functions on $\Omega$ clearly satisfies all the axioms (1.1)-(1.4).

EXAMPLE 2.8. The weighted Bergman spaces $L_{a}^{p}(\Omega, w d A)$.

The two dimensional Lebesgue area measure is denoted by $d A$. Let $w: \Omega \rightarrow \mathbf{C}$ be a continuous function with $w(\lambda)>0$ for all $\lambda \in \Omega$ and $\int_{\Omega} w(\lambda) d A(\lambda)<\infty$. For $1 \leqslant p<\infty$ the space $L_{a}^{p}(\Omega, w d A)$ consists of all analytic functions $f: \Omega \rightarrow \mathbf{C}$ with

$$
\int_{\Omega}|f(\lambda)|^{p} w(\lambda) d A(\lambda)<\infty .
$$

For $f \in L_{a}^{p}(\Omega, w d A)$ we let

$$
\|f\|_{p, w}=\left(\int_{\Omega}|f(\lambda)|^{p} w(\lambda) d A(\lambda)\right)^{1 / p} .
$$

\|\|$_{p, w}$ defines a norm on $L_{a}^{p}(\Omega, w d A)$ such that the spaces $L_{a}^{p}(\Omega, w d A)(1 \leqslant p<$ $\infty)$ are Banach spaces satisfying the axioms (1.1)-(1.4).

In fact, that $L_{a}^{p}(\Omega, w d A)$ is complete and satisfies the axioms (1.1)-(1.3) is easy to see and well known. Some information is provided in [3]. A simple estimate shows that axiom (1.4) is satisfied as well. 
If $\Omega=\mathbf{D}$ and $w(\lambda) \equiv 1$ we have the classical Bergman spaces and will write $\left(L_{a}^{p},\|\|_{p}\right)$ for $\left(L_{a}^{p}(\mathbf{D}, d A),\|\|_{p, 1}\right)$.

EXAMPLE 2.9. The spaces $D_{\alpha}(-\infty<\alpha<\infty)$ are Hilbert spaces of analytic functions satisfying the axioms (1.1)-(1.4).

Let $\Omega=\mathbf{D}$, then an analytic function $f$ can be expressed as a Taylor series, $f(z)=\sum_{n=0}^{\infty} \hat{f}(n) z^{n}$. For $\alpha \in \mathbf{R}$ we define

$$
f \in D_{\alpha} \Leftrightarrow \sum_{n=0}^{\infty}(n+1)^{\alpha}|\hat{f}(n)|^{2}<\infty .
$$

An inner product on $D_{\alpha}$ can be defined by

$$
(f, g)_{\alpha}=\sum_{n=0}^{\infty}(n+1)^{\alpha} \hat{f}(n) \overline{\hat{g}(n)} \text {. }
$$

The corresponding norm will be denoted by \|\|$_{\alpha}$.

A short survey of the known results about $D_{\alpha}$ is given in [9].

For $\alpha<0\|f\|_{\alpha}$ is equivalent to $\|f\|_{2, w}$ with $w(z)=\left(1-|z|^{2}\right)^{-1-\alpha}$ (see Example 2.8 and [27]), thus in this case $D_{\alpha}=L_{a}^{2}\left(\mathbf{D},\left(1-|z|^{2}\right)^{-1-\alpha} d A\right)$ with equivalence of norms.

For $\alpha=1$ we get the Dirichlet space $D=D_{1}$ and will write $\|f\|_{D}=\|f\|_{1}$.

For $\alpha=0$ we have the usual Hardy space $H^{2}=H^{2}(\mathbf{D})$ and will write \|\|$_{H^{2}}$ for \|\|$_{0}$.

For $0<\alpha \leqslant 1$ spaces closely related to $D_{\alpha}$ were considered by Carleson in his dissertation [11].

2.4. The Cowen-Douglas classes $\mathscr{B}_{n}(G)$. A special class of operators on Hilbert spaces was considered by M. J. Cowen and R. G. Douglas. Let $\mathscr{H}$ be a separable Hilbert space, $G$ be a connected open subset of $\mathbf{C}$ and $n$ a positive integer. Recall from [13] that an operator $T \in \mathscr{L}(\mathscr{H})$ belongs to the class $\mathscr{B}_{n}(G)$ if the following four conditions are satisfied.

$$
\begin{aligned}
& G \subseteq \sigma(T), \\
& \operatorname{ran}(T-\lambda)=\mathscr{H} \quad \text { for } \lambda \in G, \\
& \operatorname{span}\{\operatorname{ker}(T-\lambda): \lambda \in G\}=\mathscr{H}, \\
& \operatorname{dim} \operatorname{ker}(T-\lambda)=n \quad \text { for } \lambda \in G .
\end{aligned}
$$

In [13] Cowen and Douglas gave a complete set of unitary invariants for an operator in $\mathscr{B}_{1}(G)$. The following theorem shows how the class $\mathscr{B}_{1}(G)$ is related to the operators $M_{z}$ considered here.

THEOREM 2.10. Let $\Omega$ be a connected and open set in the complex plane. An operator $T \in \mathscr{L}(\mathscr{H})$ is in the class $\mathscr{B}_{1}(\bar{\Omega})$ if and only if there exists a Hilbert space $\mathscr{K} \neq\{0\}$ of analytic functions on $\Omega$ satisfying (1.1)-(1.4) such that the Hilbert space adjoint $T^{*}$ of $T$ is unitarily equivalent to $M_{z} \in \mathscr{L}(\mathscr{K})$.

Proof. Suppose $T \in \mathscr{B}_{1}(\bar{\Omega})$. We have to construct a Hilbert space $\mathscr{K}$ satisfying (1.1)-(1.4) and a unitary operator $U: \mathscr{H} \rightarrow \mathscr{K}$ such that $U T^{*}=M_{z} U$. 
We shall follow the construction of Cowen and Douglas [13,1.15] and verify that it has the required properties. (Note that the set $\Omega$ of $[13,1.15]$ corresponds to $\bar{\Omega}$ in our notation.) Using Grauert's theorem [15] Cowen and Douglas obtain a holomorphic map $\gamma: \bar{\Omega} \rightarrow \mathscr{H}$ (i.e. the map $\omega \rightarrow(\gamma(\omega), x)$ is analytic on $\bar{\Omega}$ for all $x \in \mathscr{H}$ ) which has the further property that $\gamma(\omega)$ spans $\operatorname{ker}(T-\omega)$ for all $\omega \in \bar{\Omega}$.

For every $x \in \mathscr{H}$ we can now define a function $f$ on $\Omega$ by letting $f(\lambda)=(x, \gamma(\bar{\lambda}))$. The set $\mathscr{K}=\{f: \Omega \rightarrow \mathbf{C}: f(\lambda)=(x, \gamma(\bar{\lambda}))$ for some $x \in \mathscr{H}\}$ can be made into a linear space by defining addition and scalar multiplication the obvious way. Thus $U$ : $\mathscr{H} \rightarrow \mathscr{K}, U x=f$, where $f(\lambda)=(x, \gamma(\bar{\lambda}))$, is a linear map. We shall show that $U$ is 1-1. Suppose $U x=0$ for some $x \in \mathscr{H}$, then $(x, \gamma(\omega))=0$ for all $\omega \in \bar{\Omega}$. But $\gamma(\omega)$ spans $\operatorname{ker}(T-\omega)$, so $x$ must be orthogonal to $\operatorname{span}\{\operatorname{ker}(T-\omega): \omega \in \bar{\Omega}\}=\mathscr{H}$, thus $x=0$.

We can now define an inner product on $\mathscr{K}$ by setting

$$
(f, g)_{\mathscr{K}}=\left(U^{-1} f, U^{-1} g\right)_{\mathscr{H}} \text {. }
$$

This makes $U$ into an isometry. Since $U$ is onto by definition, $\mathscr{K}$ is a Hilbert space and $U$ is a unitary operator.

It follows from the properties of $\gamma$ that $\mathscr{K}$ is contained in the space of all analytic functions on $\Omega$, hence $\mathscr{K}$ satisfies (1.1). That (1.2) is also satisfied follows from the inequality

$$
|f(\lambda)|=\left|\left(U^{-1} f, \gamma(\bar{\lambda})\right)\right| \leqslant\left\|U^{-1} f\right\|_{\mathscr{H}}\|\gamma(\bar{\lambda})\|_{\mathscr{H}}=\|f\|_{\mathscr{K}}\|\gamma(\bar{\lambda})\|_{\mathscr{H}} .
$$

To show that (1.3) is valid, we take $f \in \mathscr{K}$ and note that

$$
0=\left(U^{-1} f,(T-\omega) \gamma(\omega)\right)=\left(T^{*} U^{-1} f, \gamma(\omega)\right)-\bar{\omega}\left(U^{-1} f, \gamma(\omega)\right) \text { for } \omega \in \bar{\Omega} \text {. }
$$

Thus for $\lambda \in \Omega$ we have $\lambda f(\lambda)=\lambda\left(U^{-1} f, \gamma(\bar{\lambda})\right)=\left(T^{*} U^{-1} f, \gamma(\bar{\lambda})\right)$, which means that $z f=U T^{*} U^{-1} f \in \mathscr{K}$.

This last computation also establishes that $M_{z}=U T^{*} U^{-1}$ or $M_{z} U=U T^{*}$.

Finally, by Lemma $2.1,(1.4)$ will follow if we verify (2.3)(i), (ii), and (iii). Fix $\lambda \in \Omega$. Then the function $k_{\lambda}=U \gamma(\lambda)$ satisfies $k_{\lambda}(\lambda)=(\gamma(\lambda), \gamma(\lambda))=\|\gamma(\lambda)\|^{2} \neq$ 0 , i.e. (2.3)(i). By $(2.8)(T-\bar{\lambda})$ is onto, thus $\left(M_{z}-\lambda\right)=U\left(T^{*}-\lambda\right) U^{-1}$ is bounded below, i.e. (2.3)(ii). Similarly, we see that (2.3)(iii), $\operatorname{dim} \operatorname{ker}\left(M_{z}-\lambda\right)^{*}=1$, is true, because $\operatorname{dim} \operatorname{ker}(T-\bar{\lambda})=1$ (see (2.10)).

We shall now establish the converse. To this end suppose that $\mathscr{H}$ is a Hilbert space of analytic functions, which satisfies $(1.1)-(1.4)$. The class $\mathscr{B}_{1}(\bar{\Omega})$ is invariant under unitary equivalence. Thus it is enough to show that $M_{z}^{*} \in \mathscr{B}_{1}(\bar{\Omega})$.

We have already seen that $\Omega \subseteq \sigma\left(M_{z}\right)$, hence $\bar{\Omega} \subseteq \sigma\left(M_{z}^{*}\right)$, which is (2.7). If $\lambda \in \Omega$, then we know from Lemma 2.1 that $\left(M_{z}-\lambda\right)$ is bounded below, thus $\left(M_{z}-\lambda\right)^{*}$ is onto (2.8). To show (2.9) we take $f \in \mathscr{H}$ and assume $f \perp \operatorname{ker}\left(M_{z}-\lambda\right)^{*}$ for all $\lambda \in \Omega$. By Remark 2.2 it follows that $f \perp k_{\lambda}$ for all $\lambda \in \Omega$, i.e. $f(\lambda)=$ $\left(f, k_{\lambda}\right)=0$ and therefore $f=0$. Finally, (2.10) follows immediately from Lemma 2.1.

2.5. Invariant subspaces. If $T$ is an operator on a Banach space $\mathscr{X}$, then a (closed) subspace $\mathscr{M}$ of $\mathscr{X}$ is called invariant for $T$ if $T \mathscr{M} \subseteq \mathscr{M}$. An invariant subspace is called hyperinvariant for $T$, if it is invariant for all operators which commute with $T$. 
The collection of all invariant subspaces of an operator $T$ is denoted by Lat $T$. It forms a complete lattice with respect to intersections and closed spans. The subcollection of hyperinvariant subspaces of $T, \mathrm{H}$-Lat $T$, forms a complete sublattice of Lat $T$.

From now on suppose that $\Omega$ is a bounded, nonempty region in the complex plane and that $\mathscr{B}$ is a Banach space of analytic functions on $\Omega$ satisfying (1.1)-(1.4). By an invariant (hyperinvariant) subspace we will always mean an invariant (hyperinvariant) subspace for $\left(M_{z}, \mathscr{B}\right)$.

RemarK 2.11. It follows from the description of the commutant of $M_{z}$ (Corollary 2.5 ) that a subspace $\mathscr{M}$ is hyperinvariant if and only if $M(\mathscr{B}) \mathscr{M} \subseteq \mathscr{M}$.

For a subset $S$ of $\mathscr{B}$ we will write [S] for the smallest invariant subspace which contains all of $S$. For single functions $f \in \mathscr{B}$ we will simply write $[f]$ for $[\{f\}]$. Such invariant subspaces are called cyclic. Note that the linear manifold $\{p f: p$ is a polynomial $\}$ is dense in $[f]$. Similarly we shall write $M[S]$ (resp. $M[f]$ ) for the smallest hyperinvariant subspace (invariant under $M(\mathscr{B})$ ) which contains the set $S \subseteq \mathscr{B}$ (resp. the function $f \in \mathscr{B}$ ). Here we note that $M(\mathscr{B}) f$ is densely contained in $M[f]$.

If $f \in \mathscr{B}$, then let $Z(f)=\{\lambda \in \Omega: f(\lambda)=0\}$, and for a subset $S \subseteq \mathscr{B}$ denote by $Z(S)$ the zero set of $S$, i.e. the intersection of all the sets $Z(f)$ with $f \in S$.

We note that every invariant subspace $\mathscr{M}$ satisfies (1.1)-(1.3). Furthermore, $\left(M_{z}-\lambda\right) \mid \mathscr{M}$ is bounded below for all $\lambda \in \Omega$, since $M_{z}-\lambda$ is bounded below (Lemma 2.1). As in the proof of Lemma 2.1 it follows now from the Fredholm theory that $\operatorname{dim} \operatorname{ker}\left(\left(M_{z}-\lambda\right) \mid \mathscr{M}\right)^{*}=\operatorname{dim}(\mathscr{M} /(z-\lambda) \mathscr{M})$ does not depend on $\lambda \in \Omega$.

On the other hand, if we consider $\lambda \in \Omega, \lambda \notin Z(\mathscr{M})$, then there is an $f \in \mathscr{M}$ such that $f(\lambda) \neq 0$ and therefore $f \notin(z-\lambda) \mathscr{M}$, i.e. $\operatorname{dim}(\mathscr{M} /(z-\lambda) \mathscr{M})>0$ for any $\mathscr{M} \in \operatorname{Lat}\left(M_{z}, \mathscr{B}\right), \mathscr{M} \neq\{0\}$. We can now make the following important definitiion.

Definition 2.12. The map cod: $\operatorname{Lat}\left(M_{z}, \mathscr{B}\right) \rightarrow \mathbf{N} \cup\{\infty\}$ is defined as follows: If $\mathscr{M} \neq\{0\}$, then fix $\lambda \in \Omega$ and set $\operatorname{cod} \mathscr{M}=\operatorname{dim}(\mathscr{M} /(z-\lambda) \mathscr{M})$. If $\mathscr{M}=\{0\}$, then set $\operatorname{cod} \mathscr{M}=1$. We say that $\mathscr{M}$ has the codimension $n$ property if $\operatorname{cod} \mathscr{M}=n$.

The connection of the previous definition to the classes $\mathscr{B}_{n}(\bar{\Omega})(\S 2.4)$ is as follows. If $\mathscr{H}$ is a Hilbert space satisfying (1.1)-(1.4), $\mathscr{M} \in \operatorname{Lat}\left(M_{z}, \mathscr{H}\right), \mathscr{M} \neq\{0\}$, and $\operatorname{cod} \mathscr{M}=n<\infty$, then $\left(M_{z} \mid \mathscr{M}\right)^{*} \in \mathscr{B}_{n}(\bar{\Omega})$.

The following proposition points to the importance of the codimension one property.

Proposition 2.13. If $\mathscr{M} \in \operatorname{Lat}\left(M_{z}, \mathscr{B}\right)$ and $\operatorname{cod} \mathscr{M}=1$, then there is a Banach space $\mathscr{E}$ on $\Omega$ satisfying (1.1)-(1.4), and there exists $\varphi \in M(\mathscr{E}, \mathscr{M})$ such that $M_{\varphi}$ maps $\mathscr{E}$ isometrically onto $\mathscr{M}$ and

$$
\left(M_{z} \mid \mathscr{M}\right) M_{\varphi}=M_{\varphi}\left(M_{z}, \mathscr{E}\right) .
$$

Note that, if $\mathscr{M}$ is a subspace of a Hilbert space, then the norm on $\mathscr{E}$ is actually a Hilbert space norm. 
Proof. We may assume that $\mathscr{M} \neq\{0\}$. Then $Z(\mathscr{M})$ is a discrete subset of $\Omega$ with accumulation points only on $\partial \Omega$, say $Z(\mathscr{M})=\left\{\lambda_{n}\right\}$. Furthermore, for every $n$ there is a positive integer $m_{n}$ such that every $f \in \mathscr{M}$ has a zero of order at least $m_{n}$ at $\lambda_{n}$, but there is an $f_{n} \in \mathscr{M}$ such that $f_{n}$ does not have a zero of order $m_{n}+1$ at $\lambda_{n}$.

By a theorem of Weierstrass (see e.g. [18, Theorem 12.2]) there is an analytic function $\varphi$ on $\Omega$, which has its zeros exactly at the points $\left\{\lambda_{n}\right\}$, and the order of the zero at $\lambda_{n}$ is $m_{n}$. It follows that $f / \varphi$ is analytic on $\Omega$ for all $f \in \mathscr{M}$.

Define

$$
\mathscr{E}=\{f / \varphi: f \in \mathscr{M}\}, \quad\|f / \varphi\|_{\mathscr{E}}=\|f\|_{\mathscr{B}} .
$$

By definition $M_{\varphi}: \mathscr{E} \rightarrow \mathscr{M}$ is an isometric isomorphism onto $\mathscr{M}$.

Claim. $\mathscr{E}$ satisfies (1.1)-(1.3).

Proof. (1.1) is satisfied by the choice of $\varphi$.

(1.2) Suppose first that $\lambda \notin Z(\mathscr{M})$. Then we have $|\varphi(\lambda)| \neq 0$ and

$$
\left|\frac{f(\lambda)}{\varphi(\lambda)}\right| \leqslant \frac{c}{|\varphi(\lambda)|}\|f\|_{\mathscr{B}}=\frac{c}{|\varphi(\lambda)|}\|f / \varphi\|_{\mathscr{E}} \text {. }
$$

If $\lambda \in Z(\mathscr{M})$, then there is an $\varepsilon>0$ such that

$$
C=\left\{\lambda+\varepsilon e^{i t}: t \in[0,2 \pi)\right\} \subseteq \Omega
$$

and $C \cap Z(\mathscr{M})=\varnothing . C$ is compact, thus $\inf \{|\varphi(\zeta)|: \zeta \in C\}=c_{1}>0$. Also, the unit ball of $\mathscr{B}$ is a normal family (see Proposition 1 of [9]), hence there is a $c_{2}>0$ such that $|f(\zeta)| \leqslant c_{2}\|f\|_{\mathscr{B}}$ for all $\zeta \in C$.

We have

$$
\left|\frac{f(\lambda)}{\varphi(\lambda)}\right|=\left|\frac{1}{2 \pi i} \int_{C} \frac{f(\zeta) / \varphi(\zeta)}{\zeta-\lambda} d \zeta\right| \leqslant\left(c_{2} / c_{1}\right)\|f\|_{\mathscr{B}}=c\|f / \varphi\|_{\mathscr{E}} .
$$

(1.3) is satisfied since $\mathscr{M} \in \operatorname{Lat}\left(M_{z}, \mathscr{B}\right)$ and $M_{\varphi} M_{z}=\left(M_{z} \mid \mathscr{M}\right) M_{\varphi}$, here we wrote $M_{z}$ for $\left(M_{z}, \mathscr{E}\right)$.

The last equality establishes (2.11).

Finally, to show that $\mathscr{E}$ satisfies (1.4), we shall use Lemma 2.1 and verify (2.2) with $\lambda_{0} \notin Z(\mathscr{M})$. Thus (i) is satisfied by the choice of $\varphi$. (ii) and (iii) are satisfied since $M_{\varphi}$ is an isometric isomorphism intertwining $\left(M_{z} \mid \mathscr{M}\right)$ and $M_{z} \in \mathscr{L}(\mathscr{E})$ and $\left(M_{z} \mid \mathscr{M}\right)$ satisfies (ii) and (iii).

We are interested in the lattice structure of $\operatorname{Lat}\left(M_{z}, \mathscr{B}\right)$. To simplify the problem, we shall consider the "sublattice" of invariant subspaces with the codimension one property. Before we start, however, we shall state some results about invariant subspaces with the codimension $n$ property for arbitrary $n$.

REMARK 2.14. Beurling's theorem [6] states that every invariant subspace of $\left(M_{z}, H^{2}\right)$ is of the form $\varphi H^{2}=[\varphi]$, where $\varphi$ is an inner function. We shall see below (Corollary 3.3) that cyclic invariant subspaces always have the codimension one property, thus every invariant subspace of $\left(M_{z}, H^{2}\right)$ has the codimension one property.

Remark 2.15. Consider the spaces $D_{\alpha}, \alpha<0$, from Example 2.9. If $\mathscr{M} \in$ $\operatorname{Lat}\left(M_{z}, D_{\alpha}\right), \mathscr{M} \neq\{0\}$, and $n \in \mathbf{N} \cup\{\infty\}$, then there is an invariant subspace $\mathscr{M}_{n} \subseteq \mathscr{M}$ such that $\operatorname{cod} \mathscr{M}_{n}=n$. This follows from results of Apostol, Bercovici, 
Foiaş, and Pearcy (see Corollary 6.9 and Proposition 5.4 of [5]). Similarly it follows from results of Bercovici [4] that for every $n \in \mathbf{N} \cup\{\infty\}$ there is a hyperinvariant subspace for $\left(M_{z}, L_{a}^{2}(\Omega, d A)\right.$ ) (see Example 2.8) which has the codimension $n$ property. We note here that the proofs in [5 and 4] are of an abstract nature involving the axiom of choice. No explicit examples of functions in $D_{\alpha}(\alpha<0)$ which span an invariant subspace with the codimension $n$ property, $n \geqslant 2$, have been found.

The next proposition gives a preliminary result about the relation between the codimension $n$ property and the span of invariant subspaces. For the codimension one property we shall go into more detail later.

Proposition 2.16. Suppose $\mathscr{M}, \mathscr{N} \in \operatorname{Lat}\left(M_{z}, \mathscr{B}\right)$.

(a) We have

$$
\operatorname{cod}(\mathscr{M} \vee \mathscr{N}) \leqslant \operatorname{cod} \mathscr{M}+\operatorname{cod} \mathscr{N}
$$

(b) Furthermore, if $\operatorname{Lat}\left(M_{z}, \mathscr{B}\right)$ contains an invariant subspace with the codimension $m$ property, $m \geqslant 2$, and $n_{1}, n_{2} \in \mathbf{N} \cup\{\infty\}, n_{1}+n_{2}=m$, then there are invariant subspaces $\mathscr{N}_{1}$ and $\mathscr{N}_{2}$ such that

$$
\begin{gathered}
\operatorname{cod} \mathscr{N}_{i}=n_{i}, \quad i=1,2, \quad \text { and } \\
\operatorname{cod}\left(\mathscr{N}_{1} \vee \mathscr{N}_{2}\right)=\operatorname{cod} \mathscr{N}_{1}+\operatorname{cod} \mathscr{N}_{2} .
\end{gathered}
$$

Proof. (a) Fix $\lambda \in \Omega$. If either $\operatorname{cod} \mathscr{M}$ or $\operatorname{cod} \mathscr{N}$ is infinite, then there is nothing to prove. So we may assume that $\operatorname{cod} \mathscr{M}<\infty$ and $\operatorname{cod} \mathscr{N}<\infty$.

Thus there are finite dimensional subspaces $\mathscr{M}_{1}$ and $\mathscr{N}_{1}$ of $\mathscr{M}$ and $\mathscr{N}$ respectively such that $\mathscr{M}=(z-\lambda) \mathscr{M}+\mathscr{M}_{1}, \mathscr{N}=(z-\lambda) \mathscr{N}+\mathscr{N}_{1}, \operatorname{dim} \mathscr{M}_{1}=\operatorname{cod} \mathscr{M}$, and $\operatorname{dim} \mathscr{N}_{1}=\operatorname{cod} \mathscr{N}$. We see that

$$
\begin{aligned}
\mathscr{M}+\mathscr{N} & =(z-\lambda) \mathscr{M}+\mathscr{M}_{1}+(z-\lambda) \mathscr{N}+\mathscr{N}_{1} \\
& =(z-\lambda)(\mathscr{M}+\mathscr{N})+\mathscr{M}_{1}+\mathscr{N}_{1} \\
& \subseteq(z-\lambda)(\mathscr{M} \vee \mathscr{N})+\left(\mathscr{M}_{1}+\mathscr{N}_{1}\right) \\
& \subseteq \mathscr{M} \vee \mathscr{N} .
\end{aligned}
$$

The second to last expression is the sum of a closed and a finite dimensional subspace, hence it is closed. Since $\mathscr{M}+\mathscr{N}$ is dense in $\mathscr{M} \vee \mathscr{N}$ we obtain that the last inclusion in (2.12) is actually an equality. From this it follows that

$$
\operatorname{cod}(\mathscr{M} \vee \mathscr{N}) \leqslant \operatorname{dim}\left(\mathscr{M}_{1}+\mathscr{N}_{1}\right) \leqslant \operatorname{cod} \mathscr{M}+\operatorname{cod} \mathscr{N}
$$

(b) To see that equality in (a) can actually occur let us assume that $m \in \mathbf{N} \cup\{\infty\}$, $m \geqslant 2$, is given and that $\operatorname{Lat}\left(M_{z}, \mathscr{B}\right)$ contains an invariant subspace $\mathscr{M}$ with codimension $m$ property. First assume $m=n_{1}+n_{2}, n_{1}, n_{2} \in \mathbf{N}$. We shall construct $\mathcal{N}_{1}, \mathcal{N}_{2} \in \operatorname{Lat}\left(M_{z}, \mathscr{B}\right)$ with $\operatorname{cod} \mathscr{N}_{1}=n_{1}, \operatorname{cod} \mathscr{N}_{2}=n_{2}$, and $\operatorname{cod}\left(\mathscr{N}_{1} \vee \mathscr{N}_{2}\right)=m$ $=n_{1}+n_{2}$.

Fix $\lambda \in \Omega$. As above there is an $m$-dimensional subspace $\mathscr{M}_{1}$ of $\mathscr{M}$ such that $\mathscr{M}=(z-\lambda) \mathscr{M}+\mathscr{M}_{1}$. Let $\left\{f_{1}, \ldots, f_{m}\right\}$ be a basis for $\mathscr{M}_{1}$ and define $\mathscr{N}_{1}$ to be the smallest invariant subspace of $M_{z}$ which contains $\left\{f_{1}, \ldots, f_{n_{1}}\right\}$, define $\mathscr{N}_{2}$ to be the smallest invariant subspace which contains $\left\{f_{n_{1}+1}, \ldots, f_{m}\right\}$. It is easy to see that $\mathscr{N}_{1} \vee \mathscr{N}_{2}$ is the smallest invariant subspace of $M_{z}$ which contains $\left\{f_{1}, \ldots, f_{m}\right\}$. 
Claim. $\operatorname{cod} \mathscr{N}_{1}=n_{1}$.

Let $\mathscr{L}$ be the linear span of $\left\{f_{1}, \ldots, f_{n_{1}}\right\}$. Then $\mathscr{L} \subseteq \mathscr{M}_{1}$. We have $\mathscr{M}_{1} \cap$ $(z-\lambda) \mathscr{M}=\{0\}$, thus $(z-\lambda) \mathscr{N}_{1} \subseteq(z-\lambda) \mathscr{M}$ implies $\mathscr{L} \cap(z-\lambda) \mathscr{N}_{1}=\{0\}$. $(z-\lambda) \mathscr{N}_{1}$ is closed, $\mathscr{L}$ is finite dimensional, hence $\mathscr{L}+(z-\lambda) \mathscr{N}_{1} \subseteq \mathscr{N}_{1}$ is closed. We note that $\mathscr{L}+(z-\lambda) \mathscr{N}_{1}$ is invariant for $M_{z}$, hence by definition of $\mathscr{N}_{1}$ we have $\mathscr{L}+(z-\lambda) \mathscr{N}_{1}=\mathscr{N}_{1}$. This implies that $\operatorname{cod} \mathscr{N}_{1}=\operatorname{dim} \mathscr{L}=n_{1}$.

Similarly we see that $\operatorname{cod} \mathscr{N}_{2}=n_{2}$ and $\operatorname{cod}\left(\mathscr{N}_{1} \vee \mathscr{N}_{2}\right)=m$

Finally, if $m=\infty$ and $n_{1}+n_{2}=m$, then either $n_{1}$ or $n_{2}$ equals $\infty$. Suppose $n_{1}=\infty$. Set $\mathscr{N}_{1}=\mathscr{M}$.

If $n_{2}=\infty$, then set $\mathscr{N}_{2}=\mathscr{M}$. In this case we are done, because $\mathscr{M}=\mathscr{M} \vee \mathscr{M}$. So suppose $n_{2} \in \mathbf{N}$. Fix $\lambda \in \Omega$. Since $\operatorname{cod} \mathscr{M}=\infty$ there is an $n_{2}$-dimensional subspace $\mathscr{M}_{2}$ of $\mathscr{M}$ such that $(z-\lambda) \mathscr{M} \cap \mathscr{M}_{2}=\{0\}$. We define $\mathscr{N}_{2}$ to be the smallest invariant subspace which contains all of $\mathscr{M}_{2}$. As in the argument given above it follows that $\mathscr{N}_{2}$ has the codimension $n_{2}$ property.

Clearly $\mathscr{N}_{2} \subseteq \mathscr{M}$, thus $\mathscr{M}=\mathscr{N}_{1} \vee \mathscr{N}_{2}$.

\section{The codimension one property.}

3.1. Preliminaries. We shall now investigate when an invariant subspace has the codimension one property. We are aware of two results that are known. Both were proved only for Hilbert spaces.

In [14] M. Cowen and R. Douglas proved a theorem about operators in $\mathscr{B}_{1}(\bar{\Omega})$. By Theorem 2.10 it is equivalent to the following: If $\mathscr{H}$ is a Hilbert space of analytic functions on $\Omega$ satisfying axioms (1.1)-(1.4) and $\mathscr{M}$ is an invariant subspace of $\left(M_{z}, \mathscr{H}\right)$ which has finite codimension in $\mathscr{H}$, then $\mathscr{M}$ has the codimension one property.

To state the other result we have to recall a definition of Olin and Thomson [20]. Suppose $\mathscr{X}$ is an abstract Banach space. An operator $T \in \mathscr{L}(\mathscr{X})$ is called cellularindecomposable if $\mathscr{M} \cap \mathscr{N} \neq\{0\}$ for any two nonzero invariant subspaces $\mathscr{M}, \mathscr{N}$ of $T$. A Hilbert space result of Bourdon [7] states that if $\left(M_{z}, \mathscr{H}\right)$ is cellular-indecomposable, then every invariant subspace has the codimension one property. This result also follows from Lemma 4 of [20] or the proofs of Theorem 9.2 and Corollary 9.3 of [5]. In Corollary 3.15 we shall give a condition on $\left(M_{z}, \mathscr{B}\right)$ for $\mathscr{B}$ a Banach space of analytic functions, which will be necessary and sufficient for every invariant subspace to have the codimension one property.

The following useful lemma appeared in a less general context in [16 and 7].

Lemma 3.1. Suppose $\mathscr{M} \in \operatorname{Lat}\left(M_{z}, \mathscr{B}\right)$ and $\lambda \in \Omega \backslash Z(\mathscr{M})$. The following are equivalent:

$$
\begin{aligned}
& \operatorname{cod} \mathscr{M}=1, \\
& \text { if } f \in \mathscr{M}, f(\lambda)=0, \text { then there is a function } h \in \mathscr{M} \\
& \quad \text { such that }(z-\lambda) h=f, \\
& \text { if }(z-\lambda) h \in \mathscr{M} \text { for some } h \in \mathscr{B} \text {, then } h \in \mathscr{M} .
\end{aligned}
$$

Proof. Recall that $\mathscr{M}$ satisfies (1.1)-(1.3) and $\left(M_{z}-\lambda\right) \mid \mathscr{M}$ is bounded below for all $\lambda \in \Omega$. Thus the equivalence of (3.1) and (3.2) follows immediately from Lemma 2.1 applied to the Banach space of analytic functions $\mathscr{M}$ on the region 
$\Omega^{\prime}=\Omega \backslash Z(\mathscr{M})$. Furthermore, (3.2) implies (3.3) trivially. We shall establish the converse of this. To this end assume that (3.3) is satisfied and $f \in \mathscr{M}, f(\lambda)=0$. Then, since $\mathscr{B}$ satisfies (1.4), there is a function $h \in \mathscr{B}$ such that $(z-\lambda) h=f$. (3.3) implies that $h \in \mathscr{M}$, as was to be shown.

Note that in the previous lemma, condition (3.1) does not depend on $\lambda$, so neither do conditions (3.2) and (3.3). Furthermore, if $\mathscr{M}=\{0\}$, then (3.2) and (3.3) are trivially satisfied for all $\lambda \in \Omega$. If $\mathscr{M} \neq\{0\}$, then $Z(\mathscr{M})$ is a countable subset of $\Omega$ with accumulation points only on $\partial \Omega$, thus $\Omega \backslash Z(\mathscr{M})$ is always a nonempty open set, and one may apply the lemma.

The previous lemma and the following improvement of it will have many straightforward and important consequences. The following theorem also will be a central tool in the proof of the main result of this section.

TheOREM 3.2. Suppose $\mathscr{M} \in \operatorname{Lat}\left(M_{z}, \mathscr{B}\right)$ and $\lambda \in \Omega \backslash Z(\mathscr{M})$. The following are equivalent:

$$
\begin{aligned}
& \operatorname{cod} \mathscr{M}=1, \\
& \text { there is a linear manifold } \mathscr{L} \subseteq \mathscr{M} \text { with } \mathscr{L}^{-}=\mathscr{M} \text { such } \\
& \text { that } \lambda \notin Z(\mathscr{L}) \text { and whenever }(z-\lambda) h \in \mathscr{L} \text { for some } \\
& h \in \mathscr{B}, \text { then } h \in \mathscr{M} .
\end{aligned}
$$

Proof. (3.4) $\Rightarrow$ (3.5). Take $\mathscr{L}=\mathscr{M}$ and apply Lemma 3.1 .

$(3.5) \Rightarrow$ (3.4). We shall again use Lemma 3.1 ; in fact we will verify condition (3.3). Suppose that $h \in \mathscr{B}$ and $(z-\lambda) h \in \mathscr{M}$. We have to show that $h \in \mathscr{M}$.

Since $\mathscr{L}$ is dense in $\mathscr{M}$ we can find a sequence of functions $\left\{f_{n}\right\} \subseteq \mathscr{L}$ such that $f_{n} \rightarrow(z-\lambda) h$ as $n \rightarrow \infty$. Note that $f_{n}(\lambda) \rightarrow 0$. By assumption there is a $g \in \mathscr{L}$ such that $g(\lambda) \neq 0$. Define

$$
g_{n}=f_{n}-\left(f_{n}(\lambda) / g_{n}(\lambda)\right) g
$$

We then have $g_{n}(\lambda)=0, g_{n} \in \mathscr{L}$ for all $n$ and $g_{n} \rightarrow(z-\lambda) h$ as $n \rightarrow \infty$. Since $\mathscr{B}$ satisfies axiom (1.4) there is a sequence $\left\{h_{n}\right\} \subseteq \mathscr{B}$ such that $(z-\lambda) h_{n}=g_{n}$. We see that $(z-\lambda) h_{n} \rightarrow(z-\lambda) h . M_{z}-\lambda$ is bounded below, thus $h_{n} \rightarrow h$. By (3.5) we get that $\left\{h_{n}\right\} \subseteq \mathscr{M}$. It follows that $h \in \mathscr{M}$.

The next corollary is well known.

Corollary 3.3. Suppose $f \in \mathscr{B}$. Then we have

$$
\begin{aligned}
& \operatorname{cod}[f]=1 \quad \text { and } \\
& \operatorname{cod} M[f]=1
\end{aligned}
$$

Proof. We shall use Theorem 3.2. If $f=0$, then the result is true by definition. Hence we may assume that we can find a $\lambda \in \Omega$ such that $f(\lambda) \neq 0$. Fix such a $\lambda$.

As noted above the linear manifold $\mathscr{L}=\{p f: p$ is a polynomial $\}$ is dense in $[f]$. If $(z-\lambda) h \in \mathscr{L}$, then there is a polynomial $q$ such that $(z-\lambda) h=(z-\lambda) q f$. Thus $h=q f \in[f]$, hence $\operatorname{cod}[f]=1$ by Theorem 3.2.

To see that $\operatorname{cod} M[f]=1$ we recall that $\mathscr{L}=M(\mathscr{B}) f$ is dense in $M[f]$. If $(z-\lambda) h \in \mathscr{L}$ for some $h \in \mathscr{B}$, then there is a multiplier $\varphi$ such that $\varphi f=(z-\lambda) h$. It follows that $\varphi(\lambda)=0$. By Proposition 2.6 $M(\mathscr{B})$ satisfies axiom (1.4), thus there 
is a $\psi \in M(\mathscr{B})$ such that $(z-\lambda) \psi=\varphi$. Putting it all together we find that $(z-\lambda) h=\varphi f=(z-\lambda) \psi f$, hence $h=\psi f \in M[f]$, so $\operatorname{cod} M[f]=1$.

If $\left\{\lambda_{k}\right\}_{k \in \mathbf{N}}$ is a sequence of (not necessarily distinct) points in $\Omega$, then by $f\left(\left\{\lambda_{k}\right\}\right)=0$ we mean that if $\lambda$ occurs in $\left\{\lambda_{k}\right\}_{k \in N} n$ times, then $f$ has a zero at $\lambda$ of order at least $n$. In this case the set $\mathscr{M}=\left\{f \in \mathscr{B}: f\left(\left\{\lambda_{k}\right\}\right)=0\right\}$ defines a hyperinvariant subspace for $\left(M_{z}, \mathscr{B}\right)$. We clearly have $\left\{\lambda_{k}\right\}_{k \in \mathbf{N}} \subseteq Z(\mathscr{M})$.

Corollary 3.4. If $\left\{\lambda_{k}\right\}_{k \in N}$ is a sequence of points in $\Omega$, then $\mathscr{M}=\{f \in \mathscr{B}$ : $\left.f\left(\left\{\lambda_{k}\right\}\right)=0\right\}$ has the codimension one property.

Proof. If $\mathscr{M}=\{0\}$, then the result is true by definition. So assume that we can fix $\lambda \in \Omega \backslash Z(\mathscr{M})$. We shall verify condition (3.3) of Lemma 3.1.

Suppose $(z-\lambda) h \in \mathscr{M}$ for some $h \in \mathscr{B}$. We have to show that $h \in \mathscr{M}$, i.e. $h\left(\left\{\lambda_{k}\right\}\right)=0$. But it is clear that $((z-\lambda) h)\left(\left\{\lambda_{k}\right\}\right)=0$ if and only if $h\left(\left\{\lambda_{k}\right\}\right)=0$ since $\lambda \notin\left\{\lambda_{k}\right\}$.

3.2. Multipliers and the codimension one property. Corollary 3.3 states that invariant subspaces which contain $M(\mathscr{B}) f$ for some $f \in \mathscr{B}$ as a dense set have the codimension one property. The next proposition will show that a hyperinvariant subspace $\mathscr{M} \subseteq M[f]$ has the codimension one property if it contains only one element of the form $\varphi f, \varphi \in M(\mathscr{B})$. Before we state and prove the proposition we need a lemma.

Lemma 3.5. Suppose $f \in \mathscr{B}, \varphi \in M(\mathscr{B})$, and $h \in M[f]$. Then $\varphi h \in M[\varphi f]$.

Proof. $M(\mathscr{B}) f$ is dense in $M[f]$, thus there is a sequence $\left\{\varphi_{n}\right\} \subseteq M(\mathscr{B})$ such that $\varphi_{n} f \rightarrow h$. Multiplication by $\varphi$ is continuous, hence $\varphi \varphi_{n} f \rightarrow \varphi h$. But $\varphi \varphi_{n} f \in$ $M[\varphi f]$ for all $n$ and therefore $\varphi h \in M[\varphi f]$.

Proposition 3.6. Suppose $f \in \mathscr{B}, \mathscr{M} \in \mathrm{H}-\operatorname{Lat}\left(M_{z}, \mathscr{B}\right), \mathscr{M} \subseteq M[f]$.

If there is a nonzero multiplier $\varphi \in M(\mathscr{B})$ such that $\varphi f \in \mathscr{M}$, then

$$
\operatorname{cod} \mathscr{M}=1 \text {. }
$$

In particular, if the multipliers are densely contained in $\mathscr{B}$ and if a hyperinvariant subspace $\mathscr{M}$ contains a nonzero multiplier, then $\mathscr{M}$ has the codimension one property. This follows from the proposition, because we can choose $f(\lambda) \equiv 1 \in$ $M(\mathscr{B}) \subseteq \mathscr{B}$.

Proof. We may assume that $f \neq 0$. Suppose that $\varphi f \in \mathscr{M}, \varphi \in M(\mathscr{B})$, and $\lambda \in \Omega$ such that $\varphi(\lambda) f(\lambda) \neq 0$. To show that $\operatorname{cod} \mathscr{M}=1$ we shall again use Lemma 3.1. So assume $(z-\lambda) h \in \mathscr{M}$ for some $h \in \mathscr{B}$. We have to how that $h \in \mathscr{M}$. First note that $h \in M[f]$ by Corollary 3.3 and Lemma 3.1. By assumption $\varphi$ is a multiplier, thus $\varphi-\varphi(\lambda)$ is a multiplier. The multipliers satisfy (1.4), i.e. $(\varphi-\varphi(\lambda)) /(z-\lambda) \in M(\mathscr{B}) . \mathscr{M}$ is a hyperinvariant subspace therefore

$$
(\varphi-\varphi(\lambda)) h=\frac{\varphi-\varphi(\lambda)}{z-\lambda}(z-\lambda) h \in \mathscr{M} .
$$

From Lemma 3.5 we know that $\varphi h \in M[\varphi f] \subseteq \mathscr{M}$, this implies that $\varphi(\lambda) h \in \mathscr{M}$. But $\varphi(\lambda) \neq 0$, thus $h \in \mathscr{M}$. 
In general, Proposition 3.6 does not give a necessary condition for a hyperinvariant subspace to have the codimension one property. We shall later prove a much stronger theorem (Theorem 3.10), which will also imply Proposition 3.6. However, we decided to include the above proof anyway, because it is a simple proof of a result with many important applications, some of which are presented below.

EXAMPLE 3.7. For the Bergman spaces $\mathscr{B}=L_{a}^{p}(\Omega, w d A), p \geqslant 1$, we have $M(\mathscr{B})$ $=H^{\infty}(\Omega)$ and $H^{\infty}(\Omega) \subseteq L_{a}^{p}(\Omega, w d A)$. For many regions $\Omega$ and functions $w$ one knows that $H^{\infty}(\Omega)$ is dense in $L_{a}^{p}(\Omega, w d A)$. For example, if $\Omega$ is bounded and simply connected and $w(\lambda) \equiv 1$, then it is a theorem of Hedberg (see [25, p. 112]) that $H^{\infty}(\Omega)$ is dense in $L_{a}^{2}(\Omega, d A)$. Thus, in these cases, if a hyperinvariant subspace $\mathscr{M}$ contains a bounded analytic function, then $\operatorname{cod} \mathscr{M}=1$.

If $\Omega=\mathbf{D}$, we can strengthen the above statement about invariant subspaces of $\left(M_{z}, L_{a}^{p}(\mathbf{D}, w d A)\right)$ having the codimension one property. First note that in this case all invariant subspaces must be hyperinvariant. This follows, because the polynomials are weak* dense in $H^{\infty}(\mathbf{D})$, and the weak* convergence in $H^{\infty}(\mathbf{D})$ implies WOT convergence in $L_{a}^{p}(\mathbf{D}, w d A)$. Then recall that every function $f$ in the Nevanlinna class $N$ can be written as the quotient of two bounded analytic functions $\varphi_{1}, \varphi_{2}$, $f=\varphi_{1} / \varphi_{2}$.

So, if $H^{\infty}(\mathbf{D})$ is dense in $L_{a}^{p}(\mathbf{D}, w d A)$ and if an invariant subspace $\mathscr{M}$ of $\left(M_{z}, L_{a}^{p}(\mathbf{D}, w d A)\right)$ contains the Nevanlinna class function $f \neq 0$, then $\varphi_{2} f=\varphi_{1} \in$ $\mathscr{M}$, i.e. $\mathscr{M}$ contains a bounded function and thus has the codimension one property.

This last statement is true as well for the spaces $D_{\alpha}, \alpha<0$.

If $\mathscr{B}$ is an algebra with 1 , then $M(\mathscr{B})=\mathscr{B}$ and the hyperinvariant subspaces correspond to the closed ideals. Thus we immediately get the following corollary.

COROLlARY 3.8. If $\mathscr{B}$ is an algebra, then every closed ideal has the codimension one property.

EXAMPLE 3.9. (a) Every closed ideal of $H^{\infty}(\Omega)$ has the codimension one property. We shall see later (Example 3.11) that there are invariant subspaces of $\left(M_{z}, H^{\infty}(\mathbf{D})\right)$ which do not have the codimension one property.

(b) The spaces $D_{\alpha}, \alpha>1$, are well known to be algebras (see [25, Example 1, p. 99]). Furthermore the polynomials are dense in $D_{\alpha}$, thus every invariant subspace is an ideal, and we see that every invariant subspace of $\left(M_{z}, D_{\alpha}\right), \alpha>1$, has the codimension one property. This was observed by P. Bourdon [7]. In [23] it is shown that this result is also true for the Dirichlet space $D=D_{1}$.

3.3. Spans and the codimension one property. We now come to the central result of this section. In Corollary 3.3 we saw that cyclic invariant subspaces have the codimension one property. We know that all invariant subspaces are the span of cyclic invariant subspaces; in fact, if $\mathscr{M} \in \operatorname{Lat}\left(M_{z}, \mathscr{B}\right)$, then

$$
\mathscr{M}=\bigvee_{f \in \mathscr{M}}[f] \text {. }
$$

Thus in order to be able to say something about $\operatorname{cod} \mathscr{M}$ for arbitrary $\mathscr{M}$ we at least have to be able to determine $\operatorname{cod}([f] \vee[g])$ for arbitrary $f, g \in \mathscr{B}$. Proposition 2.16 implies that $\operatorname{cod}([f] \vee[g])$ is either one or two. The following theorem gives 
necessary and sufficient conditions on $[f]$ and $[g]$ for $[f] \vee[g]$ to have the codimension one property. In fact, the theorem applies to arbitrary invariant subspaces with the codimension one property.

THEOREM 3.10. Suppose $\mathscr{M}_{1}, \mathscr{M}_{2} \in \operatorname{Lat}\left(M_{z}, \mathscr{B}\right), \operatorname{cod} \mathscr{M}_{1}=\operatorname{cod} \mathscr{M}_{2}=1$, and $\lambda$ $\in \Omega, \lambda \notin Z\left(\mathscr{M}_{1}\right) \cup Z\left(\mathscr{M}_{2}\right)$.

The following are equivalent.

$$
\operatorname{cod}\left(\mathscr{M}_{1} \vee \mathscr{M}_{2}\right)=1,
$$

there are sequences $\left\{g_{n}^{1}\right\} \subseteq \mathscr{M}_{1},\left\{g_{n}^{2}\right\} \subseteq \mathscr{M}_{2}$ such that $g_{n}^{1}(\lambda)=g_{n}^{2}(\lambda)=1$ and $\left\|g_{n}^{1}-g_{n}^{2}\right\| \rightarrow 0$ as $n \rightarrow \infty$, there are sequences $\left\{g_{n}^{1}\right\} \subseteq \mathscr{M}_{1},\left\{g_{n}^{2}\right\} \subseteq \mathscr{M}_{2}$ such that $g_{n}^{1}(\lambda)=g_{n}^{2}(\lambda)=1$ and $g_{n}^{1}-g_{n}^{2} \rightarrow 0($ weakly $)$ as $n \rightarrow \infty$.

If $\mathscr{B}$ is a reflexive Banach space, then any one of the conditions above is equivalent to

$$
\begin{aligned}
& \text { there are sequences }\left\{g_{n}^{1}\right\} \subseteq \mathscr{M}_{1},\left\{g_{n}^{2}\right\} \subseteq \mathscr{M}_{2} \text { such that } \\
& g_{n}^{1}(\lambda)=g_{n}^{2}(\lambda)=1,\left\|g_{n}^{1}-g_{n}^{2}\right\| \leqslant M \text { for all } n \text { and }
\end{aligned}
$$$$
\text { some } M>0 \text {, and } g_{n}^{1}(z)-g_{n}^{2}(z) \rightarrow 0 \text { as } n \rightarrow \infty
$$

for all $z \in \Omega$.

Proof. (3.8) $\Rightarrow$ (3.9). Since $\lambda \notin Z\left(\mathscr{M}_{1}\right) \cup Z\left(\mathscr{M}_{2}\right)$ there are functions $f_{1} \in \mathscr{M}_{1}$ and $f_{2} \in \mathscr{M}_{2}$ with $f_{1}(\lambda)=f_{2}(\lambda)=1$. The function $f_{1}-f_{2} \in \mathscr{M}_{1} \vee \mathscr{M}_{2}$ vanishes at $\lambda$, thus there is an $h \in \mathscr{M}_{1} \vee \mathscr{M}_{2}$ such that $(z-\lambda) h=f_{1}-f_{2} . \mathscr{M}_{1}+\mathscr{M}_{2}$ is dense in $\mathscr{M}_{1} \vee \mathscr{M}_{2}$, consequently we can find sequences $\left\{h_{n}^{1}\right\} \subseteq \mathscr{M}_{1},\left\{h_{n}^{2}\right\} \subseteq \mathscr{M}_{2}$ such that $h_{n}^{1}-h_{n}^{2} \rightarrow h$ in norm. It follows that $(z-\lambda) h_{n}^{1}-(z-\lambda) h_{n}^{2} \rightarrow(z-\lambda) h$ $=f_{1}-f_{2}$. If we set $g_{n}^{1}=f_{1}-(z-\lambda) h_{n}^{1}$ and $g_{n}^{2}=f_{2}-(z-\lambda) h_{n}^{2}$, then we have $g_{n}^{1}(\lambda)=g_{n}^{2}(\lambda)=1,\left\{g_{n}^{1}\right\} \subseteq \mathscr{M}_{1},\left\{g_{n}^{2}\right\} \subseteq \mathscr{M}_{2}$ and

$$
\begin{aligned}
g_{n}^{1}-g_{n}^{2} & =\left(f_{1}-(z-\lambda) h_{n}^{1}\right)-\left(f_{2}-(z-\lambda) h_{n}^{2}\right) \\
& =\left(f_{1}-f_{2}\right)-\left((z-\lambda) h_{n}^{1}-(z-\lambda) h_{n}^{2}\right) \rightarrow 0 .
\end{aligned}
$$

Thus condition (3.9) is satisfied with the sequences $\left\{g_{n}^{1}\right\}$ and $\left\{g_{n}^{2}\right\}$.

(3.9) $\Rightarrow(3.10)$ is trivially true.

(3.10) $\Rightarrow$ (3.8). We shall verify condition (3.5) of Theorem 3.2. As the dense linear manifold we choose $\mathscr{M}_{1}+\mathscr{M}_{2} \subseteq \mathscr{M}_{1} \vee \mathscr{M}_{2}$. Suppose $(z-\lambda) h \in \mathscr{M}_{1}+\mathscr{M}_{2}$ for some $h \in \mathscr{B}$. We have to show that $h \in \mathscr{M}_{1} \vee \mathscr{M}_{2}$.

We know that there are functions $f_{1} \in \mathscr{M}_{1}$ and $f_{2} \in \mathscr{M}_{2}$ such that $(z-\lambda) h=$ $f_{1}+f_{2}$. Then

$$
\begin{aligned}
(z-\lambda) h & =f_{1}+f_{2} \\
& =f_{1}-f_{1}(\lambda) g_{n}^{1}+f_{2}-f_{2}(\lambda) g_{n}^{2}+f_{1}(\lambda) g_{n}^{1}+f_{2}(\lambda) g_{n}^{2} .
\end{aligned}
$$


Here $\left\{g_{n}^{i}\right\} \subseteq \mathscr{M}_{i}, i=1,2$, are the sequences given by condition (3.10). Hence we see that $f_{i}-f_{i}(\lambda) g_{n}^{i} \in \mathscr{M}_{i}, i=1,2$, vanishes for all $n$ at $\lambda$. $\mathscr{M}_{i}$ has the codimension one property; therefore we can find $\left\{h_{n}^{i}\right\} \subseteq \mathscr{M}_{i}$ such that $(z-\lambda) h_{n}^{i}=f_{i}-f_{i}(\lambda) g_{n}^{i}$. We also note that $f_{2}(\lambda)=-f_{1}(\lambda)$. Substituting into (3.12) we obtain

$$
(z-\lambda) h=(z-\lambda) h_{n}^{1}+(z-\lambda) h_{n}^{2}+f_{1}(\lambda)\left(g_{n}^{1}-g_{n}^{2}\right)
$$

By assumption the term on the right converges weakly to zero, so we get

$$
(z-\lambda)\left(h_{n}^{1}+h_{n}^{2}\right) \rightarrow(z-\lambda) h \text { (weakly) }
$$

The operator $\left(M_{z}-\lambda\right)$ is bounded below, this implies that $h_{n}^{1}+h_{n}^{2} \rightarrow h$ (weakly). But $\mathscr{M}_{1} \vee \mathscr{M}_{2}$ is a subspace and therefore weakly closed. It follows that $h \in \mathscr{M}_{1} \vee$ $\mathscr{M}_{2}$, as we had to show.

Finally, that (3.11) is equivalent to (3.10) if $\mathscr{B}$ is reflexive follows from Proposition 2, p. 272 of [9]. Actually, there the authors assumed that $\mathscr{B}$ is separable, an assumption that follows automatically for reflexive Banach spaces of analytic functions satisfying axioms (1.1) and (1.2).

We can now give the promised example of an invariant subspace of $\left(M_{z}, H^{\infty}(\mathbf{D})\right)$ with the codimension two property.

EXAmple 3.11. The disc algebra $A=A(\mathbf{D})$ is the norm closure of the polynomials in $H^{\infty}(\mathbf{D})$, i.e. it is an invariant subspace of $\left(M_{z}, H^{\infty}(\mathbf{D})\right)$, in fact $A=$ [1]. Suppose $\left\{z_{k}\right\}_{k \in \mathbf{N}} \subseteq \mathbf{D}$ is a sequence of points that clusters at every boundary point of $\mathbf{D}$.

Claim. $\|g\|_{H^{x}}=\sup \left\{\left|g\left(z_{k}\right)\right|: k \in \mathbf{N}\right\}$ for $g \in A$.

Proof. Clearly we have $\|g\|_{H^{\infty}} \geqslant \sup \left\{\left|g\left(z_{k}\right)\right|: k \in \mathbf{N}\right\}$.

To see that the inequality is true in the other direction as well, note that $g$ is continuous on $\mathbf{D}^{-}$. Thus using the maximum principle we see that $\sup \{|g(z)|$ : $\left.z \in \mathbf{D}^{-}\right\}$is attained at some $w \in \partial \mathbf{D}$, i.e. $|g(w)|=\|g\|_{H^{\infty}}$. By assumption there is a subsequence $\left\{z_{k}\right\}$ of $\left\{z_{k}\right\}$ such that $z_{k} \rightarrow w$ as $j \rightarrow \infty$. The continuity of $g$ implies that $\left|g\left(z_{k}\right)\right| \rightarrow\left|g\left(w^{\prime}\right)\right|$ and therefore $\|g\|_{H^{\infty}}=\sup \left\{\left|g\left(z_{k}\right)\right|: k \in \mathbf{N}\right\}$.

If $\left\{z_{k}\right\}_{k \in \mathbf{N}}$ is a Blaschke sequence which has every boundary point of $\mathbf{D}$ as a limit point, and if $b$ is a Blaschke product with zeros $\left\{z_{k}\right\}$, then $A b$ and $H^{\infty}(\mathbf{D}) b$ are closed in $H^{\infty}(\mathbf{D})$. In fact, $[b]=A b$ and $M[b]=H^{\infty}(\mathbf{D}) b$.

Claim. If $\mathscr{M}, \mathscr{N} \in \operatorname{Lat}\left(M_{z}, H^{\infty}(\mathbf{D})\right), \operatorname{cod} \mathscr{M}=\operatorname{cod} \mathscr{N}=1$, and $\{0\} \neq \mathscr{M} \subseteq A,\{0\}$ $\neq \mathscr{N} \subseteq M[b]$, then $\operatorname{cod}(\mathscr{M} \vee \mathscr{N})=2$.

Proof. Let $g \in \mathscr{M} \subseteq A, f \in \mathscr{N} \subseteq H^{\infty}(\mathbf{D}) b$, then

$$
\begin{aligned}
\|g-f\|_{H^{x}} & =\sup _{z \in \mathbf{D}}|g(z)-f(z)| \geqslant \sup _{k \in \mathbf{N}}\left|g\left(z_{k}\right)-f\left(z_{k}\right)\right| \\
& =\sup _{k \in \mathbf{N}}\left|g\left(z_{k}\right)\right|=\|g\|_{H^{\infty}} .
\end{aligned}
$$

Thus if $\lambda \in \mathbf{D}$ such that $g(\lambda)=1$, then

$$
\|g-f\|_{H^{x}} \geqslant\|g\|_{H^{x}} \geqslant|g(\lambda)|=1 .
$$

By condition (3.9) of Theorem 3.10 and Proposition 2.16 this implies that $\operatorname{cod}(\mathscr{M} \vee \mathscr{N})=2$. 
Corollary 3.12. Suppose $\mathscr{M}_{1}, \mathscr{M}_{2} \in \operatorname{Lat}\left(M_{z}, \mathscr{B}\right), \operatorname{cod} \mathscr{M}_{1}=\operatorname{cod} \mathscr{M}_{2}=1$. If $\mathscr{M}_{1} \cap \mathscr{M}_{2} \neq\{0\}$, then $\operatorname{cod}\left(\mathscr{M}_{1} \vee \mathscr{M}_{2}\right)=1$.

Proof. If $\mathscr{M}_{1} \cap \mathscr{M}_{2} \neq\{0\}$, then there is a function $g \in \mathscr{M}_{1} \cap \mathscr{M}_{2}$ and a $\lambda \in \Omega$ such that $g(\lambda)=1$. We can now apply Theorem 3.10 with the sequences given by $g_{n}^{1}=g_{n}^{2}=g$ for all $n \in \mathbf{N}$.

Before giving some more examples to illustrate Theorem 3.10 and Corollary 3.12 we shall prove a theorem which will enable us to apply those theorems to a more general class of invariant subspaces. Theorem 3.10 says that $\mathscr{M}_{1} \vee \mathscr{M}_{2}$ has the codimension one property, if $\mathscr{M}_{1}$ and $\mathscr{M}_{2}$ are "close" to one another in some sense. Part (a) of the next theorem is a consequence of that observation.

Theorem 3.13. (a) Suppose $\mathscr{M}, \mathscr{N}, \mathscr{L} \in \operatorname{Lat}\left(M_{z}, \mathscr{B}\right), \operatorname{cod} \mathscr{M}=\operatorname{cod} \mathscr{N}=\operatorname{cod} \mathscr{L}=$ 1 , and $\{0\} \neq \mathscr{M} \subseteq \mathscr{N}$.

If $\operatorname{cod}(\mathscr{M} \vee \mathscr{L})=1$, then $\operatorname{cod}(\mathscr{N} \vee \mathscr{L})=1$.

(b) Suppose $\left\{\mathscr{M}_{\gamma}\right\}_{\gamma \in \Gamma}$ is a family of invariant subspaces with the codimension one property and $\mathscr{M}_{\gamma_{0}} \in\left\{\mathscr{M}_{\gamma}\right\}_{\gamma \in \Gamma}, \mathscr{M}_{\gamma_{0}} \neq\{0\}$.

If $\operatorname{cod}\left(\mathscr{M}_{\gamma_{0}} \vee \mathscr{M}_{\gamma}\right)=1$ for all $\gamma \in \Gamma$, then $\mathscr{M}=\bigvee_{\gamma \in \Gamma} \mathscr{M}_{\gamma}$ has the codimension one property.

Proof. (a) If $\mathscr{L}=\{0\}$, then the result is trivial. So assume there is a $\lambda \in \Omega$, $\lambda \notin Z(\mathscr{M}) \cup Z(\mathscr{L})$. The assertion now follows immediately from (3.9) of Theorem 3.10, because we assumed $\mathscr{M} \subseteq \mathscr{N}$.

(b) Choose $\lambda \in \Omega \backslash Z\left(\mathscr{M}_{\gamma_{0}}\right)$. We shall use Theorem 3.2 and verify (3.5) with the linear manifold $\mathscr{L}$ which consists of all finite linear combinations of functions in $\mathscr{M}_{\gamma}, \gamma \in \Gamma$. To this end suppose that $(z-\lambda) h \in \mathscr{L}$ for some $h \in \mathscr{B}$. We have to show that $h \in \mathscr{M}$.

By definition of $\mathscr{L}(z-\lambda) h$ is contained in the span $\mathscr{N}$ of a finite subfamily of $\left\{\mathscr{M}_{\gamma}\right\}_{\gamma \in \Gamma}$. A repeated application of (a) shows that $\mathscr{M}_{\gamma_{0}} \vee \mathscr{N}$ has the codimension one property. Furthermore $\lambda \notin Z\left(\mathscr{M}_{\gamma_{0}} \vee \mathscr{N}\right)$, hence $h \in \mathscr{M}_{\gamma_{0}} \vee \mathscr{N} \subseteq \mathscr{M}$ by Lemma 3.1 .

In the next theorem we denote for $r \geqslant 1$ by $M[S]_{r}$ (resp. $[S]_{r}$ ) the smallest hyperinvariant (resp. invariant) subspace of $L_{a}^{r}(\Omega, w d A)$ which contains the set $S \subseteq L_{a}^{r}(\Omega, w d A)$. Note that for $r \leqslant q M[S]_{r} \cap L_{a}^{q}(\Omega, w d A)$ is a (closed) hyperinvariant subspace of $\left(M_{z}, L_{a}^{q}(\Omega, w d A)\right)$ since $L_{a}^{q}(\Omega, w d A) \subseteq L_{a}^{r}(\Omega, w d A)$ under our assumptions on $\Omega$ and $w$ (see Example 2.8).

THEOREM 3.14. Suppose $1 / p+1 / q=1 / r, p \geqslant q \geqslant r \geqslant 1$, and that $H^{\infty}(\Omega)$ is dense in $L_{a}^{s}(\Omega, w d A)$ for all $s \geqslant q$.

If $S \subseteq L_{a}^{q}(\Omega, w d A)$ and $S \cap L_{a}^{p}(\Omega, w d A) \neq\{0\}$, then

$$
\begin{gathered}
\operatorname{cod}\left(M[S]_{r}\right)=1, \\
\operatorname{cod}\left(M[S]_{r} \cap L_{a}^{q}(\Omega, w d A)\right)=1 .
\end{gathered}
$$

In particular, if $p \geqslant 2, \mathscr{M} \in \mathrm{H}-\operatorname{Lat}\left(M_{z}, L_{a}^{p}(\Omega, w d A)\right)$, then $[\mathscr{M}]_{p / 2}$ and $[\mathscr{M}]_{p / 2} \cap L_{a}^{p}(\Omega, w d A)$ have the codimension one property. 
Proof. Suppose $f \in S \cap L_{a}^{p}(\Omega, w d A), f \neq 0$.

(a) By Theorem (3.13)(b) we have to show that $M[f]_{r} \vee M[g]_{r}$ has the codimension one property for all $g \in S$. We shall show that $M[f]_{r} \cap M[g]_{r} \neq\{0\}$, whenever $g \neq 0$. This will prove part (a) by Corollary 3.12 and (3.7) of Corollary 3.3.

Claim. If $g \in S$, then $f g \in M[f]_{r} \cap M[g]_{r}$.

Suppose $\left\{\varphi_{n}\right\},\left\{\psi_{n}\right\} \subseteq H^{\infty}(\Omega)$ such that $\varphi_{n} \rightarrow f$ in $L_{a}^{p}(\Omega, w d A), \psi_{n} \rightarrow g$ in $L_{a}^{q}(\Omega, w d A)$, then $f g \in L_{a}^{r}(\Omega, w d A)$ and

$$
\left\|\varphi_{n} g-f g\right\|_{r} \leqslant\|g\|_{q}\left\|\varphi_{n}-f\right\|_{p}, \quad\left\|\psi_{n} f-f g\right\|_{r} \leqslant\|f\|_{p}\left\|\psi_{n}-g\right\|_{q}
$$

by the generalized Hölder inequality. Thus $\varphi_{n} g \rightarrow f g$ and $\psi_{n} f \rightarrow f g$ in $L_{a}^{r}(\Omega, w d A)$. $M\left(L_{a}^{r}(\Omega, w d A)\right)=H^{\infty}(\Omega)$ implies that $\varphi_{n} g \in M[g]_{r}$ and $\psi_{n} f \in M[f]_{r}$ for all $n \in \mathbf{N}$, i.e. $f g \in M[f]_{r} \cap M[g]_{r}$.

(b) This will follow from (a) and Lemma 3.1. Fix $\lambda \in \Omega$ such that $f(\lambda) \neq 0$ (i.e. $\left.\lambda \notin Z\left(M[S]_{r} \cap L_{a}^{q}(\Omega, w d A)\right)\right)$ and assume that $(z-\lambda) h \in M[S]_{r} \cap L_{a}^{q}(\Omega, w d A)$ for some $h \in L_{a}^{q}(\Omega, w d A)$. We have to show that $h \in M[S]_{r} \cap L_{a}^{q}(\Omega, w d A)$. But by (a) and Lemma $3.1 h \in M[S]_{r}$, by assumption $h \in L_{a}^{q}(\Omega, w d A)$, thus we are done.

The proof of Theorem 3.14 does not use the full strength of Theorem 3.10, it only uses Corollary 3.12. Thus, it seems likely that a better theorem than 3.14 is true.

One can prove a similar theorem for the spaces $D_{\alpha}, \alpha<0$ (see [21]).

As a special case, Theorem 3.10 gives necessary and sufficient conditions for the span of two cyclic invariant subspaces to have the codimension one property. We can use that together with Theorem 3.13(b) to give a necessary and sufficient condition so that every invariant subspace has the codimension one property.

COROllaRY 3.15. Every invariant subspace of $\left(M_{z}, \mathscr{B}\right)$ has the codimension one property if and only if the span of any two cyclic invariant subspaces has the codimension one property. (For a condition see Theorem 3.10.)

Proof. It is clear that the span of any two cyclic invariant subspaces has to have the codimension one property. The reverse implication follows from Theorem 3.13(b), because as mentioned above we have $\mathscr{M}=\mathrm{V}_{f \in \mathscr{M}}[f]$.

It follows from Corollary 3.12 that if $\left(M_{z}, \mathscr{B}\right)$ is cellular-indecomposable then every invariant subspace has the codimension one property.

3.4. The lattice of invariant subspaces with the codimension one property. Theorem 3.13 tells us when spans have the codimension one property. In the next theorem we shall see that intersections of invariant subspaces with the codimension one property always have the codimension one property. Afterwards we shall use this for an interesting construction.

THEOREM 3.16. Suppose $\left\{\mathscr{M}_{\gamma}\right\}_{\gamma \in \Gamma}$ is a family of invariant subspaces with the codimension one property.

Then $\mathscr{M}=\bigcap_{\gamma \in \Gamma} \mathscr{M}_{\gamma}$ has the codimension one property.

Proof. If $\mathscr{M}=\{0\}$, then the result is true by definition. So suppose we can choose $\lambda \in \Omega \backslash Z(\mathscr{M})$. We shall use Lemma 3.1. Suppose $(z-\lambda) h \in \mathscr{M}$ for some $h \in \mathscr{B}$. We have to show that $h \in \mathscr{M}$. 
For a fixed $\gamma \in \Gamma$ we have $\lambda \in \Omega \backslash Z\left(\mathscr{M}_{\gamma}\right),(z-\lambda) h \in \mathscr{M}_{\gamma}, \operatorname{cod} \mathscr{M}_{\gamma}=1$, thus $h \in \mathscr{M}_{\gamma}$. Hence $h \in \mathscr{M}=\bigcap_{\gamma \in \Gamma} \mathscr{M}_{\gamma}$.

Definition 3.17. Suppose $\mathscr{M} \in \operatorname{Lat}\left(M_{z}, \mathscr{B}\right)$.

(a) $I(\mathscr{M}):=\bigcap_{\gamma} N_{\gamma}$, the intersection is taken over all invariant subspaces $\mathscr{N}_{\gamma}$ with $\mathscr{M} \subseteq \mathscr{N}_{\gamma}$ and $\operatorname{cod} \mathscr{N}_{\gamma}=1$

(b) If $\left\{\mathscr{M}_{\gamma}\right\}_{\gamma \in \Gamma}$ is any family of invariant subspaces,

$$
\operatorname{sp}\left(\left\{\mathscr{M}_{\gamma}\right\}_{\gamma \in \Gamma}\right):=I\left(\bigvee_{\gamma \in \Gamma} \mathscr{M}_{\gamma}\right) .
$$

Note that we have $I(\mathscr{M}) \in \operatorname{Lat}\left(M_{z}, \mathscr{B}\right)$ and $\operatorname{cod}(I(\mathscr{M}))=1$, thus $I(\mathscr{M})$ is the smallest invariant subspace of $\left(M_{z}, \mathscr{B}\right)$ which contains $\mathscr{M}$ and has the codimension one property.

Proposition 3.18. The invariant subspaces with codimension one property form a complete lattice with respect to $\cap$ and $\mathrm{sp}$.

Proof. We have to check several things. Suppose $\mathscr{M}, \mathscr{N}, \mathscr{L} \in \operatorname{Lat}\left(M_{z}, \mathscr{B}\right)$ with $\operatorname{cod} \mathscr{M}=\operatorname{cod} \mathscr{N}=\operatorname{cod} \mathscr{L}=1$. Theorem 3.16 and the remark preceding Proposition 3.18 imply that $\mathscr{M} \cap \mathscr{N}$ and $\operatorname{sp}(\mathscr{M}, \mathscr{N})$ again have the codimension one property. The lattice axioms which involve only intersections are satisfied, because $\operatorname{Lat}\left(M_{z}, \mathscr{B}\right)$ forms a lattice with respect to $\cap$ and $\vee$. We shall verify the axioms involving sp.

(a) Claim. $\mathscr{M} \cap \operatorname{sp}(\mathscr{M}, \mathscr{N})=\mathscr{M}$.

$\mathscr{M} \cap \operatorname{sp}(\mathscr{M}, \mathscr{N}) \subseteq \mathscr{M}$ is clear.

$\mathscr{M} \subseteq \mathscr{M} \vee \mathscr{N} \Rightarrow \mathscr{M} \subseteq \operatorname{sp}(\mathscr{M}, \mathscr{N}) \Rightarrow \mathscr{M} \subseteq \mathscr{M} \cap \operatorname{sp}(\mathscr{M}, \mathscr{N})$.

(b) Claim. $\operatorname{sp}(\mathscr{M}, \mathscr{N})=\operatorname{sp}(\mathscr{N}, \mathscr{M})$.

This is trivial.

(c) Claim. $\operatorname{sp}(\operatorname{sp}(\mathscr{M}, \mathscr{N}), \mathscr{L})=\operatorname{sp}(\mathscr{M}, \operatorname{sp}(\mathscr{N}, \mathscr{L}))$.

We shall show $\operatorname{sp}(\operatorname{sp}(\mathscr{M}, \mathscr{N}), \mathscr{L})=I(\mathscr{M} \vee \mathscr{N} \vee \mathscr{L})$. This will imply the claim, because we then have

$$
\begin{aligned}
\operatorname{sp}(\mathscr{M}, \operatorname{sp}(\mathscr{N}, \mathscr{L})) & =\operatorname{sp}(\operatorname{sp}(\mathscr{N}, \mathscr{L}), \mathscr{M})=I(\mathscr{N} \vee \mathscr{L} \vee \mathscr{M}) \\
& =I(\mathscr{M} \vee \mathscr{N} \vee \mathscr{L})=\operatorname{sp}(\operatorname{sp}(\mathscr{M}, \mathscr{N}), \mathscr{L}) .
\end{aligned}
$$

Clearly $\mathscr{M} \vee \mathscr{N} \vee \mathscr{L} \subseteq \operatorname{sp}(\mathscr{M}, \mathscr{N}) \vee \mathscr{L}$, thus

$$
I(\mathscr{M} \vee \mathscr{N} \vee \mathscr{L}) \subseteq I(\operatorname{sp}(\mathscr{M}, \mathscr{N}) \vee \mathscr{L})=\operatorname{sp}(\operatorname{sp}(\mathscr{M}, \mathscr{N}), \mathscr{L})
$$

On the other hand $\mathscr{M} \vee \mathscr{N} \subseteq \mathscr{M} \vee \mathscr{N} \vee \mathscr{L}, \mathscr{L} \subseteq \mathscr{M} \vee \mathscr{N} \vee \mathscr{L} \subseteq I(\mathscr{M} \vee \mathscr{N} \vee \mathscr{L})$ Therefore $\operatorname{sp}(\mathscr{M}, \mathscr{N}) \subseteq I(\mathscr{M} \vee \mathscr{N} \vee \mathscr{L})$ and $\operatorname{sp}(\mathscr{M}, \mathscr{N}) \vee \mathscr{L} \subseteq I(\mathscr{M} \vee \mathscr{N} \vee \mathscr{L})$, thus

$$
\operatorname{sp}(\operatorname{sp}(\mathscr{M}, \mathscr{N}), \mathscr{L})=I(\operatorname{sp}(\mathscr{M}, \mathscr{N}) \vee \mathscr{L}) \subseteq I(\mathscr{M} \vee \mathscr{N} \vee \mathscr{L})
$$

(d) Claim. $\operatorname{sp}(\mathscr{M}, \mathscr{M} \cap \mathscr{N})=\mathscr{M}$.

$$
\operatorname{sp}(\mathscr{M}, \mathscr{M} \cap \mathscr{N})=I(\mathscr{M} \vee(\mathscr{M} \cap \mathscr{N}))=I(\mathscr{M})=\mathscr{M} \text {. }
$$

Finally, the lattice is complete, because we can apply the lattice operations to infinite families and stay in the class of invariant subspaces with the codimension one property (see Theorem 3.16 and Definition 3.17(b)). 
Definition 3.17 and Proposition 3.18 give rise to several questions.

Question 3.19. If $\mathscr{M} \in \operatorname{Lat}\left(M_{z}, \mathscr{B}\right)$, what are the connections between $\mathscr{M}$ and $I(\mathscr{M}) ?$

To be more specific, fix $p \geqslant 2$ and consider the spaces $L_{a}^{p}=L_{a}^{p}(\mathbf{D}, d A)$. For an invariant subspace $\mathscr{M}$ of $\left(M_{z}, L_{a}^{p}\right)$ and $1 \leqslant q \leqslant p$ we write $\mathscr{M}^{q}$ for $[\mathscr{M}]_{q} \cap L_{a}^{p}$. It follows from Theorem 3.14 that

$$
\mathscr{M}=\mathscr{M}^{p} \subseteq I(\mathscr{M}) \subseteq \mathscr{M}^{p / 2} \text { for } p \geqslant 2
$$

On the other hand we also have the inclusions

$$
\mathscr{M}=\mathscr{M}^{p} \subseteq \mathscr{M}^{q} \subseteq \mathscr{M}^{p / 2} \quad \forall p \geqslant q \geqslant p / 2 .
$$

If $\operatorname{cod} \mathscr{M} \neq 1$, then it follows that $\mathscr{M}^{p} \neq \mathscr{M}^{q}$ for some (and maybe all) $q, p>q \geqslant$ $p / 2$. This raises the question, whether in this case $\mathscr{M}^{q} \neq \mathscr{M}^{r}$ for some $q \neq r$, $q, r<p$ or $\mathscr{M}^{q}=\mathscr{M}^{r}$ for all $q, r<p$. If the last statement is true, then that would be some evidence that the answer to the following question is yes.

QUESTION 3.20. With the notations of the preceding paragraph is $I(\mathscr{M})=\mathscr{M}^{q}$ for all $q<p ?$

One result in this direction can be found in [8]. It follows from the theorem there that, if $f \in L_{a}^{p}$ and $[f]_{s}=L_{a}^{s}$ for some $0<s<p$, then $[f]_{q}=L_{a}^{q}$ for all $q<p$. Thus, in this very special case we see the following: If $\mathscr{M}=[f]_{p}$, then $\mathscr{M}^{q}=L_{a}^{p}$ for all $q<p$, thus $\mathscr{M}^{q}=\mathscr{M}^{r}$ for all $q, r<p$.

Of course here we have $\operatorname{cod} \mathscr{M}^{p}=\operatorname{cod} \mathscr{M}^{q}=1$ for all $q<p$. We also remark that the proof of this result heavily uses the fact that $f$ has no zeros in $\mathbf{D}$, i.e. $Z(\mathscr{M})=\varnothing$.

\section{Spectra associated with invariant subspaces.}

4.1. Introduction. In $\S \S 2$ and 3 we did not make any maximality assumptions on $\Omega$. However, it is clear that any serious study of a space of analytic functions will have to take into account the behavior of the functions near the boundary of the domain of holomorphy. In this article we are studying the operator $M_{z}$ and its lattice of invariant subspaces; thus it would be nice if we had a good maximality condition on $\Omega$ which does not depend on the particular representation of $\mathscr{B}$.

For example, suppose $\Omega^{\prime} \subseteq \Omega, \Omega^{\prime} \neq \varnothing, \varphi \in \operatorname{Hol}\left(\Omega^{\prime}\right), \varphi \notin \operatorname{Hol}(\Omega)$, and $\mathscr{B}$ is a Banach space of analytic functions on $\Omega$ satisfying (1.1)-(1.4). We can define a Banach space of analytic functions on $\Omega^{\prime}$ by setting

$$
\mathscr{B}^{\prime}=\left\{g: \Omega^{\prime} \rightarrow \mathbf{C}: g=\varphi f \text { for some } f \in \mathscr{B}\right\},
$$

$\|g\|_{\mathscr{B}}=\|g / \varphi\|_{\mathscr{B}}$. Then $\mathscr{B}^{\prime}$ satisfies (1.1)-(1.4) on $\Omega^{\prime} \backslash Z(\varphi)$ and $M_{\varphi}: \mathscr{B} \rightarrow \mathscr{B}^{\prime}$ is an isometric isomorphism with $M_{z}^{\prime} M_{\varphi}=M_{\varphi} M_{z}$ (see the proof of Proposition 2.13). We see that the domains of holomorphy of $\mathscr{B}$ and $\mathscr{B}^{\prime}$ differ, but the operator theoretic properties of $\left(M_{z}, \mathscr{B}\right)$ and $\left(M_{z}^{\prime}, \mathscr{B}^{\prime}\right)$ are the same.

One possibility for avoiding this problem is to try to find a "canonical representation" for $\mathscr{B}$ and then assume that for every boundary point of $\Omega$ there is a function in $\mathscr{B}$ which has a singularity at this point (compare [9]). We do not know whether it is always possible to find a suitable "canonical representation." However, 
in $\$ 5$ we shall give a positive answer under some extra assumption. See Theorem 5.3 and the remark after its proof.

Another possibility for putting a maximality condition on $\Omega$ is to consider the spectrum of the operator $\left(M_{z}, \mathscr{B}\right)$. It does not depend on the particular representation of $\mathscr{B}$, and we have seen above that we always have $\Omega^{-} \subseteq \sigma\left(M_{z}\right)$. Thus a natural assumption on $\Omega$ is $\sigma\left(M_{z}\right)=\Omega^{-}$. Under this (and some other) assumption on $\Omega$ we shall establish another type of result about invariant subspaces with the codimension one property in this section.

In [19] it was shown that for an invariant subspace $\mathscr{M}$ of $\left(M_{z}, H^{2}\right)$,

$$
\sigma_{\text {ap }}\left(M_{z}^{*} \mid \mathscr{M}^{\perp}\right)=\sigma\left(M_{z}^{*} \mid \mathscr{M}^{\perp}\right)=\{\bar{\lambda}: \varphi(\lambda)=0\}^{-} \cup \overline{\operatorname{supp}\{\mu\}},
$$

where $\varphi$ is the inner function such that $\varphi H^{2}=\mathscr{M}$ and $\mu$ is the singular measure associated with $\varphi$. In [12] this idea was generalized and used to study the invariant subspaces of $H^{2}\left(\mathbf{D}^{n}\right)\left(\mathbf{D}^{n}=\left\{\left(\lambda_{1}, \ldots, \lambda_{n}\right):\left|\lambda_{i}\right|<1\right\}\right)$. Cowen and Rubel's idea led J. Janas [16] to investigate $\sigma_{\text {ap }}\left(M_{z}^{*} \mid \mathscr{M}^{\perp}\right)$ for invariant subspaces $\mathscr{M}$ of the Bergman shift $\left(M_{z}, L_{a}^{2}\right)$. On the other hand S. Walsh [28] determined $\sigma\left(M_{z}^{*} \mid \mathscr{M}^{\perp}\right)$ for various invariant subspaces of $\left(M_{z}, L_{a}^{2}\right)$.

We shall consider $\sigma\left(M_{z}^{*} \mid \mathscr{M}^{\perp}\right)$ and $\sigma_{\text {ap }}\left(M_{z}^{*} \mid \mathscr{M}^{\perp}\right)$ for Banach spaces of analytic functions satisfying axioms (1.1)-(1.4), prove some basic results about these sets (analogous to results in [16 and 28]), and then study the relationship between $\sigma\left(M_{z}^{*} \mid \mathscr{M}^{\perp}\right)$ and $\sigma_{\text {ap }}\left(M_{z}^{*} \mid \mathscr{M}^{\perp}\right)$. It will turn out (Corollary 4.6) that, if $\sigma\left(M_{z}\right)=\Omega^{-}$ and $C \backslash \Omega^{-}$has only one component, then $\sigma\left(M_{z}^{*} \mid \mathscr{M}^{\perp}\right)=\sigma_{\text {ap }}\left(M_{z}^{*} \mid \mathscr{M}^{\perp}\right)$ if and only if $\operatorname{cod} \mathscr{M}=1$. From this we shall derive another necessary condition for an invariant subspace to have the codimension one property (see Corollary 4.8). We then apply this corollary to the Bergman spaces $L_{a}^{p}$ and obtain a result which does not follow from Theorem 3.14.

4.2. Definitions and basic results. From now on assume again that $\Omega$ is a bounded region in the complex plane and $\mathscr{B}$ is a Banach space of analytic functions on $\Omega$ satisfying (1.1)-(1.4).

By $\mathscr{M}^{\perp}$ we denote the annihilator of $\mathscr{M}$ in $\mathscr{B}^{*}$,

$$
\mathscr{M}^{\perp}=\left\{x \in \mathscr{B}^{*}:\langle f, x\rangle=0 \forall f \in \mathscr{M}\right\} .
$$

If $\mathscr{M} \in \operatorname{Lat}\left(M_{z}, \mathscr{B}\right)$, then $\mathscr{M}^{\perp} \in \operatorname{Lat}\left(M_{z}^{*}, \mathscr{B}^{*}\right)$. Thus we can make the following definition.

Definition 4.1. If $\mathscr{M} \in \operatorname{Lat}\left(M_{z}, \mathscr{B}\right)$, then

$$
\begin{aligned}
\sigma(\mathscr{M}) & =\sigma_{\text {ap }}\left(M_{z}^{*} \mid \mathscr{M}^{\perp}\right) \\
& =\left\{\lambda \in \mathbf{C}:\left(M_{z}-\lambda\right)^{*} \mid \mathscr{M}^{\perp} \text { is not bounded below }\right\} .
\end{aligned}
$$

In this definition and in the rest of this section we shall follow the convention that an operator on a zero dimensional space has an empty spectrum and an empty approximate point spectrum. Thus $\sigma\left(M_{z}^{*} \mid \mathscr{B}^{\perp}\right)=\sigma(\mathscr{B})=\varnothing$. This will be convenient for the statement of our theorems and not lead to any contradictions.

Note that we used the Banach space adjoint of $M_{z}$ to define $\sigma(\mathscr{M})$, thus our results will differ from the above-mentioned Hilbert space results by a complex conjugate. Furthermore, it will be apparent from Theorem 4.5, why we defined $\sigma(\mathscr{M})$ to be $\sigma_{\text {ap }}\left(M_{z}^{*} \mid \mathscr{M}^{\perp}\right)$ rather than $\sigma\left(M_{z}^{*} \mid \mathscr{M}^{\perp}\right)$. 
In our study of $M_{z}^{*} \mid \mathscr{M}^{\perp}$ we shall need the following lemma. Before we state the lemma we recall that $\mathscr{M}^{\perp}$ is weak* closed and can be regarded as the dual of $\mathscr{B} / \mathscr{M}$. The relative weak* topology on $\mathscr{M}^{\perp}$ regarded as a subspace of $\mathscr{B}^{*}$ coincides with the weak* topology that $\mathscr{M}^{\perp}$ obtains as the dual of $\mathscr{B} / \mathscr{M}$ (see e.g. Corollary 2.4 of $[10])$.

Lemma 4.2. Suppose $\mathscr{X}$ is a Banach space, $T \in \mathscr{L}(\mathscr{X})$ has closed range, and $\mathscr{M} \in$ Lat $T$. If $\operatorname{dim} \operatorname{ker} T^{*}<\infty$ and $\operatorname{ker} T^{*} \cap \mathscr{M}^{\perp}=\{0\}$, then $T^{*} \mathscr{M}^{\perp}$ is weak ${ }^{*}$ closed in $\mathscr{M}^{\perp}$.

Proof. It follows from the closed range theorem and the assumption that $T$ has closed range that $T^{*}$ has a closed range as well. This, together with the assumption that $\operatorname{ker} T^{*}$ is finite dimensional allows us to use Lemma IV.5.29 of [17, p. 239], and conclude that $T^{*} \mathscr{M}^{\perp}$ is norm closed in $\mathscr{M}^{\perp}$. Furthermore, $T^{*}$ is weak* continuous, hence $T^{*} \mid \mathscr{M}^{\perp}$ is weak continuous as well. We assumed that $\operatorname{ker}\left(T^{*} \mid \mathscr{M}^{\perp}\right)=$ $\{0\}$, thus $T^{*} \mid \mathscr{M}^{\perp} \in \mathscr{L}\left(\mathscr{M}^{\perp}\right)$ is a weak* continuous linear map with trivial kernel and norm closed range, by Theorem 2.7 of [10] $T^{*} \mid \mathscr{M}^{\perp}$ is weak closed in $\mathscr{M}^{\perp}$.

The next two propositions contain some straightforward results concerning $\sigma(\mathscr{M})$ (for $\left(M_{z}, L_{a}^{2}\right)$ some of these were presented in [16]).

Proposition 4.3. Suppose $\mathscr{M}, \mathscr{N} \in \operatorname{Lat}\left(M_{z}, \mathscr{B}\right)$ and $\left\{\mathscr{M}_{\gamma}\right\}_{\gamma \in \Gamma}$ is a family of invariant subspaces of $\left(M_{z}, \mathscr{B}\right)$.

Set $\mathscr{L}_{1}=\bigvee_{\gamma \in \Gamma} \mathscr{M}_{\gamma}$ and $\mathscr{L}_{2}=\bigcap_{\gamma} \mathscr{M}_{\gamma}$.

(a) $\mathscr{M} \subseteq \mathscr{N} \Rightarrow \sigma(\mathscr{N}) \subseteq \sigma(\mathscr{M})$,

(b) $\sigma\left(\mathscr{L}_{1}\right) \subseteq \cap_{\gamma} \sigma\left(\mathscr{M}_{\gamma}\right)$,

(c) $\bigcup_{\gamma} \sigma\left(\mathscr{M}_{\gamma}\right) \subseteq \sigma\left(\mathscr{L}_{2}\right)$.

ProOf. (a) $\mathscr{M} \subseteq \mathscr{N} \Rightarrow \mathscr{N}^{\perp} \subseteq \mathscr{M}^{\perp} \Rightarrow \sigma(\mathscr{N})=\sigma_{\text {ap }}\left(M_{z}^{*} \mid \mathscr{N}^{\perp}\right) \subseteq \sigma_{\text {ap }}\left(M_{z}^{*} \mid \mathscr{M}^{\perp}\right)$ $=\sigma(\mathscr{M})$.

(b) For a fixed $\gamma \in \Gamma$ we have $\mathscr{M}_{\gamma} \subseteq \mathscr{L}_{1}$, thus by (a) we see that $\sigma\left(\mathscr{L}_{1}\right) \subseteq \sigma\left(\mathscr{M}_{\gamma}\right)$ for all $\gamma \in \Gamma$.

(c) For a fixed $\gamma \in \Gamma$ we have $\mathscr{L}_{2} \subseteq \mathscr{M}_{\gamma}$, thus by (a) we see that $\sigma\left(\mathscr{M}_{\gamma}\right) \subseteq \sigma\left(\mathscr{L}_{2}\right)$ for all $\gamma \in \Gamma$.

Proposition 4.4. Let $\mathscr{M} \in \operatorname{Lat}\left(M_{z}, \mathscr{B}\right)$. Then $\sigma(\mathscr{M}) \cap \Omega=Z(\mathscr{M})$.

Proof. We shall first show that $Z(\mathscr{M}) \subseteq \sigma(\mathscr{M}) \cap \Omega$. To this end assume that $\lambda \in Z(\mathscr{M})$. By definition we know that $\lambda \in \Omega$, thus we only have to show that $\lambda \in \sigma(\mathscr{M})$. Recall from Remark 2.2 that $k_{\lambda}$ denotes the element in $\mathscr{B}^{*}$ which satisfies $f(\lambda)=\left\langle f, k_{\lambda}\right\rangle$ for all $f \in \mathscr{B}$. It follows that $0=f(\lambda)=\left\langle f, k_{\lambda}\right\rangle$ for all $f \in \mathscr{M}$, i.e. $k_{\lambda} \in \mathscr{M}^{\perp}$. Hence $k_{\lambda} \in \operatorname{ker}\left(\left(M_{z}-\lambda\right)^{*} \mid \mathscr{M}^{\perp}\right), k_{\lambda} \neq 0$, therefore $\lambda \in$ $\sigma_{\text {ap }}\left(M_{z}^{*} \mid \mathscr{M}^{\perp}\right)=\sigma(\mathscr{M})$.

Secondly we have to show that $\sigma(\mathscr{M}) \cap \Omega \subseteq Z(\mathscr{M})$. Assume $\lambda \in \Omega \backslash Z(\mathscr{M})$, we shall show that $\lambda \notin \sigma(\mathscr{M}) . \lambda \notin Z(\mathscr{M})$ implies that $k_{\lambda} \notin \mathscr{M}^{\perp}$. But $k_{\lambda}$ spans $\operatorname{ker}\left(M_{z}-\lambda\right)^{*}$, thus $\mathscr{M}^{\perp} \cap \operatorname{ker}\left(M_{z}-\lambda\right)^{*}=\{0\}$. It follows now from Lemma 4.2 that the range of $\left(M_{z}-\lambda\right)^{*} \mid \mathscr{M}^{\perp}$ is norm closed in $\mathscr{M}^{\perp}$. Together with 
$\operatorname{ker}\left(\left(M_{z}-\lambda\right)^{*} \mid \mathscr{M}^{\perp}\right)=\{0\}$ this implies that $\left(M_{z}-\lambda\right)^{*} \mid \mathscr{M}^{\perp}$ is bounded below, hence $\lambda \notin \sigma(\mathscr{M})$.

4.3. $\sigma(\mathscr{M})$ versus $\sigma\left(M_{z}^{*} \mid \mathscr{M}^{\perp}\right)$.

We are now ready to present some results which are new even for $\left(M_{z}, L_{a}^{2}\right)$. The codimension one property plays an important role in the comparison between $\sigma(\mathscr{M})$ and $\sigma\left(M_{z}^{*} \mid \mathscr{M}^{\perp}\right)$.

TheOREM 4.5. Suppose $\mathscr{M} \in \operatorname{Lat}\left(M_{z}, \mathscr{B}\right)$.

(a) If $\operatorname{cod} \mathscr{M}=1$, then $\sigma\left(M_{z}^{*} \mid \mathscr{M}^{\perp}\right) \cap \Omega=\sigma(\mathscr{M}) \cap \Omega=Z(\mathscr{M})$.

(b) If $\operatorname{cod} \mathscr{M} \neq 1$, then $\Omega^{-} \subseteq \sigma\left(M_{z}^{*} \mid \mathscr{M}^{\perp}\right)$.

Proof. (a) Assume cod $\mathscr{M}=1$. We have to show that $\sigma\left(M_{z}^{*} \mid \mathscr{M}^{\perp}\right) \cap \Omega \subseteq$ $\sigma_{\text {ap }}\left(M_{z}^{*} \mid \mathscr{M}^{\perp}\right) \cap \Omega$. To this end, assume that $\lambda \in \Omega \backslash \sigma_{\text {ap }}\left(M_{z}^{*} \mid \mathscr{M}^{\perp}\right)$, i.e. $\left(M_{z}-\lambda\right)^{*} \mid \mathscr{M}^{\perp}$ is bounded below. We have to show that $\left(M_{z}-\lambda\right)^{*} \mid \mathscr{M}^{\perp}$ maps $\mathscr{M}^{\perp}$ onto $\mathscr{M}^{\perp}$.

$\left(M_{z}-\lambda\right)^{*} \mid \mathscr{M}^{\perp}$ is bounded below; in particular we have $\operatorname{ker}\left(M_{z}-\lambda\right)^{*} \cap \mathscr{M}^{\perp}$ $=\{0\}$. It follows from Lemma 4.2 that the range of $\left(M_{z}-\lambda\right)^{*} \mid \mathscr{M}^{\perp}$ is weak* closed in $\mathscr{M}^{\perp}$. Hence it suffices to show that $\operatorname{ran}\left(\left(M_{z}-\lambda\right)^{*} \mid \mathscr{M}^{\perp}\right)$ is weak* dense in $\mathscr{M}^{\perp}$.

Suppose $\varphi$ is a weak* continuous linear functional on $\mathscr{M}^{\perp}$ which satisfies $\varphi\left(\left(M_{z}-\lambda\right)^{*} x\right)=0$ for all $x \in \mathscr{M}^{\perp}$. The conclusion in (a) will follow, if we show that $\varphi=0$. We can find an $f \in \mathscr{B}$ such that $\varphi(x)=\langle f, x\rangle$ for all $x \in \mathscr{M}^{\perp}$. From the discussion preceding Lemma 4.2, we know that $\varphi=0$ if and only if $f \in \mathscr{M} . f$ satisfies

$$
0=\varphi\left(\left(M_{z}-\lambda\right)^{*} x\right)=\left\langle f,\left(M_{z}-\lambda\right)^{*} x\right\rangle=\left\langle\left(M_{z}-\lambda\right) f, x\right\rangle \quad \forall x \in \mathscr{M}^{\perp} .
$$

Thus $(z-\lambda) f \in{ }^{\perp}\left(\mathscr{M}^{\perp}\right)=\mathscr{M}$ since $\mathscr{M}$ is norm closed. We assumed that $\lambda \in$ $\Omega \backslash \sigma(\mathscr{M})$, hence $\lambda \in \Omega \backslash Z(\mathscr{M})$ by Proposition 4.4. $\mathscr{M}$ has the codimension one property, thus Lemma 3.1 implies that $f \in \mathscr{M}$, as we had to show.

(b) Assume now that $\operatorname{cod} \mathscr{M} \neq 1$. It is enough to show that $\Omega \subseteq \sigma\left(M_{z}^{*} \mid \mathscr{M}^{\perp}\right)$, because $\sigma\left(M_{z}^{*} \mid \mathscr{M}^{\perp}\right)$ is closed. Furthermore, if $\lambda \in Z(\mathscr{M})$, then $\lambda \in \sigma(\mathscr{M}) \subseteq$ $\sigma\left(M_{z}^{*} \mid \mathscr{M}^{\perp}\right)$ by Proposition 4.4. So suppose that $\lambda \in \Omega \backslash Z(\mathscr{M})$. It follows from Lemma 3.1 that there is a function $f \in \mathscr{B}$ such that $(z-\lambda) f \in \mathscr{M}$, but $f \notin \mathscr{M}$.

We shall show that the weak* continuous linear functional $\varphi$ on $\mathscr{M}^{\perp}$ induced by $f$ annihilates the range of $\left(M_{z}-\lambda\right)^{*} \mid \mathscr{M}^{\perp} . f \notin \mathscr{M}$ implies $\varphi \neq 0$, thus it will follow that $\left(M_{z}-\lambda\right)^{*} \mid \mathscr{M}^{\perp}$ does not map $\mathscr{M}^{\perp}$ onto $\mathscr{M}^{\perp}$, hence $\lambda \in \sigma\left(M_{z}^{*} \mid \mathscr{M}^{\perp}\right)$.

For $x \in \mathscr{M}^{\perp}$ we have

$$
\varphi\left(\left(M_{z}-\lambda\right)^{*} x\right)=\left\langle f,\left(M_{z}-\lambda\right)^{*} x\right\rangle=\left\langle\left(M_{z}-\lambda\right) f, x\right\rangle=0,
$$

because $(z-\lambda) f \in \mathscr{M}$.

We do not know whether $\operatorname{cod} \mathscr{M}=1$ always implies that $\sigma(\mathscr{M})=\sigma\left(M_{z}^{*} \mid \mathscr{M}^{\perp}\right)$. We can show that under some extra assumptions on $\Omega$ this assertion is true.

Corollary 4.6. Suppose $\mathbf{C} \backslash\left(\Omega^{-}\right)$has only one component and $\sigma\left(M_{z}\right)=\Omega^{-}$.

(a) If $\mathscr{M} \in \operatorname{Lat}\left(M_{z}, \mathscr{B}\right)$ such that $\operatorname{cod} \mathscr{M}=1$, then

$$
\sigma\left(M_{z}^{*} \mid \mathscr{M}^{\perp}\right)=\sigma(\mathscr{M}) .
$$


(b) If $\mathscr{M} \in \operatorname{Lat}\left(M_{z}, \mathscr{B}\right)$ such that $\operatorname{cod} \mathscr{M} \neq 1$, then

$$
\begin{gathered}
\sigma\left(M_{z}^{*} \mid \mathscr{M}^{\perp}\right)=\Omega^{-} \text {and } \\
Z(\mathscr{M}) \cup \partial\left(\Omega^{-}\right) \subseteq \sigma(\mathscr{M}) \subseteq Z(\mathscr{M}) \cup \partial \Omega .
\end{gathered}
$$

For the proof we shall need a lemma.

Lemma 4.7. Suppose $T \in \mathscr{L}(\mathscr{X}), \mathscr{N} \in$ Lat $T$. If $C$ denotes the unbounded component of $\mathbf{C} \backslash \sigma(T)$, then $\sigma(T \mid \mathscr{N}) \cap C=\varnothing$.

Proof. For an operator on a Banach space we always have $\partial \sigma(T) \subseteq \sigma_{\text {ap }}(T)$. Thus $\partial \sigma(T \mid \mathcal{N}) \subseteq \sigma_{\text {ap }}(T \mid \mathcal{N}) \subseteq \sigma(T)$. Note that $C$ is an open and connected subset of $\mathbf{C}$, hence it is pathwise connected. So if $\lambda \in C$, then there is a path in $C$ connecting $\lambda$ and $\infty$. If $\lambda$ were also contained in $\sigma(T \mid \mathscr{N})$, then there had to be a point on the path which is also in $\partial \sigma(T \mid \mathscr{N}) \subseteq \sigma(T)$, a contradiction.

Thus $\sigma(T \mid \mathcal{N}) \cap C=\varnothing$.

Proof (OF Corollary 4.6). We have $\sigma\left(M_{z}\right)=\sigma\left(M_{z}^{*}\right)=\Omega^{-}$. Thus it follows from the assumptions that $\mathbf{C} \backslash \sigma\left(M_{z}^{*}\right)$ has only one component, the unbounded one. From Lemma 4.7 it follows that $\sigma\left(M_{z}^{*} \mid \mathscr{M}^{\perp}\right) \cap \mathbf{C} \backslash \sigma\left(M_{z}^{*}\right)=\varnothing$, thus

$$
\sigma(\mathscr{M}) \subseteq \sigma\left(M_{z}^{*} \mid \mathscr{M}^{\perp}\right) \subseteq \sigma\left(M_{z}^{*}\right)=\Omega^{-} .
$$

(a) If $\operatorname{cod} \mathscr{M}=1$, then it follows from Theorem 4.5 (a) that $\sigma(\mathscr{M}) \cap \Omega=$ $\sigma\left(M_{z}^{*} \mid \mathscr{M}^{\perp}\right) \cap \Omega$. Thus we need to show that $\sigma\left(M_{z}^{*} \mid \mathscr{M}^{\perp}\right) \cap \partial \Omega \subseteq \sigma(\mathscr{M})$.

To this end, suppose that $\lambda \in \sigma\left(M_{z}^{*} \mid \mathscr{M}^{\perp}\right) \cap \partial \Omega$. We distinguish two cases:

(1) $\lambda \in \partial \sigma\left(M_{z}^{*} \mid \mathscr{M}^{\perp}\right) \cap \partial \Omega \Rightarrow \lambda \in \sigma_{\text {ap }}\left(M_{z}^{*} \mid \mathscr{M}^{\perp}\right)=\sigma(\mathscr{M})$.

(2) $\lambda \in \partial \Omega$ and $\lambda$ is in the interior of $\sigma\left(M_{z}^{*} \mid \mathscr{M}^{\perp}\right)$. In this case there is a sequence $\left\{\lambda_{n}\right\}_{n \in \mathbf{N}} \subseteq \Omega \cap \sigma\left(M_{z}^{*} \mid \mathscr{M}^{\perp}\right)$ such that $\lambda_{n} \rightarrow \lambda$ as $n \rightarrow \infty$. It follows from Theorem 4.5 that $\left\{\lambda_{n}\right\}_{n \in \mathbf{N}} \subseteq \sigma(\mathscr{M})$. $\sigma(\mathscr{M})$ is closed, hence $\lambda \in \sigma(\mathscr{M})$.

(b) If $\operatorname{cod} \mathscr{M} \neq 1$, then Theorem 4.5(b) implies that $\Omega^{-} \subseteq \sigma\left(M_{z}^{*} \mid \mathscr{M}^{\perp}\right)$. Thus (4.4) implies $\sigma\left(M_{z}^{*} \mid \mathscr{M}^{\perp}\right)=\Omega^{-}$, which is (4.2).

To establish (4.3) we note that (4.2) implies that $\partial\left(\Omega^{-}\right)=\partial \sigma\left(M_{z}^{*} \mid \mathscr{M}^{\perp}\right) \subseteq$ $\sigma_{\text {ap }}\left(M_{z}^{*} \mid \mathscr{M}^{\perp}\right)=\sigma(\mathscr{M})$.

Hence $Z(\mathscr{M}) \cup \partial\left(\Omega^{-}\right) \subseteq \sigma(\mathscr{M})$ follows from Proposition 4.4.

On the other hand we have $\sigma(\mathscr{M}) \subseteq \Omega^{-}$, thus

$$
\sigma(\mathscr{M})=(\sigma(\mathscr{M}) \cap \Omega) \cup(\sigma(\mathscr{M}) \cap \partial \Omega) \subseteq Z(\mathscr{M}) \cup \partial \Omega .
$$

In $\S 3$ we saw that two invariant subspaces have to be "far away" from each other in order to span an invariant subspace with the codimension two property. The next corollary says that under some extra assumptions an invariant subspace which is large enough is never contained in an invariant subspace with the codimension two property. "Large enough" will mean that $\sigma(\mathscr{M})$ does not contain $\partial \Omega$.

Corollary 4.8. Suppose $\mathbf{C} \backslash\left(\Omega^{-}\right)$has only one component, $\partial\left(\Omega^{-}\right)=\partial \Omega$, and $\sigma\left(M_{z}\right)=\Omega$.

If $\mathscr{M}, \mathscr{N} \in \operatorname{Lat}\left(M_{z}, \mathscr{B}\right), \operatorname{cod} \mathscr{N} \neq 1, \operatorname{cod} \mathscr{M}=1$, and $\mathscr{M} \subseteq \mathscr{N}$ then

$$
\sigma\left(M_{z}^{*} \mid \mathscr{M}^{\perp}\right)=\sigma(\mathscr{M})=Z(\mathscr{M}) \cup \partial \Omega .
$$


Proof. $\mathscr{M}$ has the codimension one property, thus the first equality follows from Corollary 4.6(a). On the other hand, it follows from Proposition 4.3(a), Corollary 4.6(b), and the assumptions that

$$
Z(\mathscr{N}) \cup \partial \Omega=\sigma(\mathscr{N}) \subseteq \sigma(\mathscr{M}) \subseteq \Omega \cup \partial \Omega .
$$

Of course, we know that $Z(\mathscr{M}) \subseteq \sigma(\mathscr{M})$, hence

$$
Z(\mathscr{M}) \cup \partial \Omega \subseteq \sigma(\mathscr{M}) \subseteq(\sigma(\mathscr{M}) \cap \Omega) \cup(\sigma(\mathscr{M}) \cap \partial \Omega) \subseteq Z(\mathscr{M}) \cup \partial \Omega .
$$

We remark here that the assumption that $C \backslash\left(\Omega^{-}\right)$has only one component may be relaxed, if one only considers hyperinvariant subspaces.

We shall now illustrate Corollary 4.8 with an example. Suppose $\left\{\lambda_{k}\right\}_{k \in \mathbf{N}} \subseteq \mathbf{D}$ is a sequence of distinct points. For $1 \leqslant p<\infty$ we define a linear transformation from $L_{a}^{p}$ into the space of all sequences of complex numbers by

$$
T_{p} f=\left\{f\left(\lambda_{k}\right)\left(1-\left|\lambda_{k}\right|^{2}\right)^{2 / p}\right\}_{k \in \mathrm{N}} \quad \forall f \in L_{a}^{p} .
$$

Recall from [1] that the sequence $\left\{\lambda_{k}\right\}_{k \in \mathbf{N}}$ is called a universal interpolating sequence for $L_{a}^{p}$, if $T_{p}$ maps $L_{a}^{p}$ into and onto $l^{p}$, the space of all complex valued absolutely $p$-summable sequences.

Proposition 4.9. Suppose that $\left\{\lambda_{k}\right\}_{k \in \mathbf{N}}$ is a universal interpolating sequence for $L_{a}^{p}, 1 \leqslant p<\infty$.

If $\mathscr{M}=\left\{f \in L_{a}^{p}: f\left(\left\{\lambda_{k}\right\}\right)=0\right\}$, then

$$
\sigma\left(M_{z}^{*} \mid \mathscr{M}^{\perp}\right)=\sigma(\mathscr{M})=\left\{\lambda_{k}\right\}_{k \in \mathbf{N}}^{-}
$$

Proof. The first equality follows from Corollary 4.6(a) and Corollary 3.4. Proposition 4.4 implies that $\left\{\lambda_{k}\right\}_{k} \in \mathbf{N} \subseteq \sigma(\mathscr{M})$. Thus we only have to show that $\sigma(\mathscr{M}) \subseteq\left\{\lambda_{k}\right\}_{k \in \mathbf{N}}^{-}$

To this end suppose that $\lambda \notin\left\{\lambda_{k}\right\}_{k}^{-} \in \mathbf{N}$, i.e. there is a $\delta>0$ such that $\left|\lambda-\lambda_{k}\right|>\delta$ for all $k \in \mathbf{N}$. We have to show that $\left(M_{z}-\lambda\right)^{*} \mid \mathscr{M}^{\perp}$ is bounded below.

As before we denote by $k_{\mu}$ the functional of evaluation at $\mu$ :

$$
f(\mu)=\left\langle f, k_{\mu}\right\rangle \quad \forall f \in L_{a}^{p} .
$$

Since $\left\{\lambda_{n}\right\}_{n \in \mathrm{N}}$ is a universal interpolating sequence, the operator $T_{p}: L_{a}^{p} \rightarrow l^{p}$ is onto. Hence its adjoint $T_{p}^{*}: l^{q} \rightarrow\left(L_{a}^{p}\right)^{*}$ is bounded below. Here $q$ is the conjugate exponent to $p$ satisfying $1 / p+1 / q=1$, if $1<p<\infty$, and $q=\infty$, if $p=1$. We have

$$
\mathscr{M}^{\perp}=\left(\operatorname{ker} T_{p}\right)^{\perp}=\operatorname{ran} T_{p}^{*}
$$

Thus $T_{p}^{*}$ maps $l^{q}$ onto $\mathscr{M}^{\perp}$ in a 1-1 manner. Let $\left\{e_{n}\right\}_{n \in \mathrm{N}}$ be the canonical basis for $l^{q}$. We compute for $f \in L_{a}^{p}$ and $n \in \mathbf{N}$

$$
\left\langle f, T_{p}^{*} e_{n}\right\rangle=\left\langle T_{p} f, e_{n}\right\rangle=f\left(\lambda_{n}\right)\left(1-\left|\lambda_{n}\right|^{2}\right)^{2 / p}=\left\langle f,\left(1-\left|\lambda_{n}\right|^{2}\right)^{2 / p} k_{\lambda_{n}}\right\rangle .
$$

This shows that if $\left\{a_{n}\right\}_{n \in \mathbf{N}} \in l^{q}$, then

$$
T_{p}^{*}\left(\left\{a_{n}\right\}\right)=\sum_{n=1}^{\infty} a_{n}\left(1-\left|\lambda_{n}\right|^{2}\right)^{2 / p} k_{\lambda_{n}} .
$$


In the following one will have to substitute the $l^{\infty}$ norm, whenever $p=1$ and $q=\infty$. We shall not do this here.

Since $T_{p}^{*}$ is bounded below there are $K_{1}, K_{2}$ such that for all $\left\{a_{n}\right\}_{n \in \mathrm{N}} \in l^{q}$

$$
\begin{aligned}
K_{1}\left(\sum_{n=1}^{\infty}\left|a_{n}\right|^{q}\right)^{1 / q} & \leqslant\left\|\sum_{n=1}^{\infty} a_{n} k_{\lambda_{n}}\left(1-\left|\lambda_{n}\right|^{2}\right)^{2 / p}\right\|_{*} \\
& \leqslant K_{2}\left(\sum_{n=1}^{\infty}\left|a_{n}\right|^{q}\right)^{1 / q},
\end{aligned}
$$

here \|\|$_{*}$ is the norm on $\left(L_{a}^{p}\right)^{*}$.

Now, if $x \in \mathscr{M}^{\perp}=\operatorname{ran} T_{p}^{*}$, then there is a sequence $\left\{a_{n}\right\}_{n \in \mathbf{N}} \in l^{q}$ such that

$$
x=\sum_{n=1}^{\infty} a_{n} k_{\lambda_{n}}\left(1-\left|\lambda_{n}\right|^{2}\right)^{2 / p} .
$$

For such an $x$ we have

$$
\begin{aligned}
\left\|\left(M_{z}-\lambda\right)^{*} x\right\|_{*} & =\left\|\left(M_{z}-\lambda\right)^{*} \sum_{n=1}^{\infty} a_{n} k_{\lambda_{n}}\left(1-\left|\lambda_{n}\right|^{2}\right)^{2 / p}\right\|_{*} \\
& =\left\|\sum_{n=1}^{\infty} a_{n}\left(M_{z}-\lambda\right)^{*} k_{\lambda_{n}}\left(1-\left|\lambda_{n}\right|^{2}\right)^{2 / p}\right\|_{*} \\
& =\left\|\sum_{n=1}^{\infty} a_{n}\left(\lambda_{n}-\lambda\right) k_{\lambda_{n}}\left(1-\left|\lambda_{n}\right|^{2}\right)^{2 / p}\right\|_{*} \\
& \geqslant K_{1}\left(\sum_{n=1}^{\infty}\left|a_{n}\left(\lambda_{n}-\lambda\right)\right|^{q}\right)^{1 / q} \geqslant K_{1} \delta\left(\sum_{n=1}^{\infty}\left|a_{n}\right|^{q}\right)^{1 / q} \\
& \geqslant\left(\frac{K_{1}}{K_{2}}\right) \delta\left\|\sum_{n=1}^{\infty} a_{n} k_{\lambda_{n}}\left(1-\left|\lambda_{n}\right|^{2}\right)^{2 / p}\right\|_{*}=\left(\frac{K_{1}}{K_{2}}\right) \delta\left\|^{x}\right\|_{*},
\end{aligned}
$$

i.e. $\left(M_{z}-\lambda\right)^{*} \mid \mathscr{M}^{\perp}$ is bounded below as was to be shown.

Note that the idea for the above proof came from computations which can be found in [1, Lemma 1.1.1] or [2, Theorem 3.12]. However, there it was used that $L_{a}^{q}$, $1<p, q<\infty, 1 / p+1 / q=1$, can be identified with $\left(L_{a}^{p}\right)^{*}$. For our purposes this would have complicated matters since that duality is not isometric. This is the reason why we decided to repeat part of the arguments from [1 and 2].

Now recall that a sequence $\left\{\lambda_{n}\right\}_{n \in \mathbf{N}} \subseteq \mathbf{D}$ is called separated if

$$
\inf \left\{\left|\left(\lambda_{n}-\lambda_{m}\right) /\left(1-\bar{\lambda}_{n} \lambda_{m}\right)\right|: n \neq m\right\}>0 \text {. }
$$

Let $\left\{\lambda_{n}\right\}_{n \in \mathbf{N}} \subseteq \mathbf{D}$ be a sequence with the following properties:

(a) $\left\{\lambda_{n}\right\}_{n \in \mathrm{N}}$ is separated,

(b) $\left\{\lambda_{n}\right\}_{n \in \mathrm{N}}$ is not a Blaschke sequence,

(c) the closure of $\left\{\lambda_{n}\right\}_{n \in N}$ does not contain the unit circle.

It is easy to see that such sequences exist. Fix $1<p<\infty$. E. Amar [1] has shown that every separated sequence is a finite union of sequences which are universal interpolating for $L_{a}^{p}$ (note here that $p>1$ ). Thus, there is a subsequence $\left\{\mu_{k}\right\}_{k \in N}$ of $\left\{\lambda_{n}\right\}_{n \in N}$ which is universal interpolating for $L_{a}^{p}$ but not a Blaschke sequence. 
We set

$$
\mathscr{M}=\left\{f \in L_{a}^{p}: f\left(\left\{\mu_{k}\right\}\right)=0\right\} .
$$

$\mathscr{M}$ is invariant for $\left(M_{z}, L_{a}^{p}\right)$, and it follows from Proposition 4.9 and (c) that $\sigma(\mathscr{M})$ does not contain the whole unit circle. Thus Corollary 4.8 implies that every $\mathscr{N} \in \operatorname{Lat}\left(M_{z}, L_{a}^{p}\right)$ with $\mathscr{M} \subseteq \mathcal{N}$ has the codimension one property.

On the other hand we see from the construction of $\mathscr{M}$ that $\mathscr{M}$ does not contain any nonzero $H^{\infty}$ function. This shows that we would not have been able to deduce this result from Proposition 3.6 or Theorem 3.14.

We shall conclude this section with a remark about the connection between an invariant subspace $\mathscr{M}, I(\mathscr{M})$, and $\sigma(\mathscr{M})$. Recall from Definition 3.17 that $I(\mathscr{M})$ denotes the smallest invariant subspace, which has the codimension one property and contains $\mathscr{M}$.

Proposition 4.10. Suppose $\mathscr{M} \in \operatorname{Lat}\left(M_{z}, \mathscr{B}\right)$.

(a) $\sigma(I(\mathscr{M})) \subseteq \sigma(\mathscr{M})$,

(b) $\sigma(I(\mathscr{M})) \cap \Omega=Z(\mathscr{M})=\sigma(\mathscr{M}) \cap \Omega$.

Proof. (a) By definition we have $\mathscr{M} \subseteq I(\mathscr{M})$, thus (a) follows from Proposition 4.3(a).

(b) The second equality follows from Proposition 4.4. The first equality will follow, if we show that $Z(I(\mathscr{M}))=Z(\mathscr{M})$. The inclusion $\mathscr{M} \subseteq I(\mathscr{M})$ implies $Z(I(\mathscr{M})) \subseteq Z(\mathscr{M})$. So suppose $\lambda \in Z(\mathscr{M})$. Then all functions in $\mathscr{M}$ have a zero at $\lambda$, thus $\mathscr{M} \subseteq(z-\lambda) \mathscr{B}$ by axiom (1.4). But $(z-\lambda) \mathscr{B}$ has the codimension one property, hence $I(\mathscr{M}) \subseteq(z-\lambda) \mathscr{B}$. It follows that $\lambda \in Z((z-\lambda) \mathscr{B}) \subseteq Z(I(\mathscr{M}))$.

We do not know whether one always has equality in part (a) of the last proposition.

\section{Hilbert spaces of analytic functions on the unit disc.}

5.1. Introduction. Corollary 3.3 stated that cyclic invariant subspaces have the codimension one property. Hilbert spaces of analytic functions are spanned by the kernel functions $k_{\lambda}, \lambda \in \Omega$. It follows that such a Hilbert space is separable, so one might ask whether every invariant subspace of $\left(M_{z}, D_{\alpha}\right), \alpha \in \mathbf{R}$ (see Example 2.9), which has the codimension one property, is cyclic. We do not know. A related question which we can answer in certain situations arises as follows.

If $f \in \mathscr{H}$, where $\mathscr{H}$ is a Hilbert space satisfying (1.1)-(1.4) with $\Omega=\mathbf{D}$, then $g / f$ is analytic on $\mathbf{D}$ for all $g \in[f]$. Thus one might wonder, whether an invariant subspace $\mathscr{M}$ of $\left(M_{z}, \mathscr{H}\right)$ with $\operatorname{cod} \mathscr{M}=1$ contains a function with no extra zeros, i.e. a function $f \in \mathscr{M}$ such that $g / f$ is analytic on $\mathbf{D}$ for al! $g \in \mathscr{M}$. We shall indicate a method which may help to solve this problem. We shall then use this method to show that for $\left(M_{z}, D_{\alpha}\right), \alpha \geqslant 0$, the answer to the above problem is indeed yes. Using that result we shall show that for every invariant subspace $\mathscr{M}$ of $\left(M_{z}, D\right)$ ( $D$ is the Dirichlet space) there is a function $f_{0} \in \mathscr{M}$ such that $\left[f_{0}\right] \subseteq \mathscr{M} \subseteq H^{2} f_{0} \cap D$.

5.2. Left inverses of $M_{z}$. In this section we shall assume that $\mathscr{H}$ is a Hilbert space satisfying (1.1)-(1.4) with $\Omega=\mathbf{D}$ and $\sigma\left(M_{z}\right)=\mathbf{D}^{-}$. If $\mathscr{M}$ is an invariant subspace of $\left(M_{z}, \mathscr{H}\right)$ with the codimension one property, then $\left(\left(M_{z} \mid \mathscr{M}\right), \mathscr{M}\right)$ is unitarily 
equivalent to $\left(M_{z}, \mathscr{K}\right)$, where $\mathscr{K}$ satisfies (1.1)-(1.4) (see Proposition 2.13). Thus the following results about $\left(M_{z}, \mathscr{H}\right)$ will help us in finding corresponding results about $\left(\left(M_{z} \mid \mathscr{M}\right), \mathscr{M}\right)$.

The next two propositions were motivated by the proof of Proposition 1.11 of [13].

$M_{z}$ is bounded below, thus $M_{z}^{*} M_{z}$ is invertible. We set $S_{0}:=\left(M_{z}^{*} M_{z}\right)^{-1} M_{z}^{*}$. Also, for $f, g \in \mathscr{H}$ we let $f \otimes g$ denote the rank one operator on $\mathscr{H}$ that is defined by

$$
(f \otimes g)(h)=(h, g) f \text {. }
$$

Furthermore, for $\lambda \in \mathbf{D}$ we shall write $k_{\lambda}$ for the element in $\operatorname{ker}\left(M_{z}-\lambda\right)^{*}=$ $\mathscr{H} \ominus(z-\lambda) \mathscr{H}$ which satisfies $f(\lambda)=\left(f, k_{\lambda}\right)$ for all $f \in \mathscr{H}$.

Proposition 5.1. Suppose $\Omega=\mathbf{D}, \sigma\left(M_{z}\right)=\mathbf{D}^{-}$.

(a) If $g \in \mathscr{H}, g(0)=1$, then $S=S_{0}\left(1-g \otimes k_{0}\right)$ is a left inverse of $M_{z}$ with $g \in \operatorname{ker} S$.

(b) If $S$ is a left inverse of $M_{z}$, then there is exactly one $g \in \operatorname{ker} S$ with $g(0)=1$.

Proof. (a) We compute

$$
\begin{aligned}
S M_{z} & =S_{0}\left(1-g \otimes k_{0}\right) M_{z}=S_{0} M_{z}-S_{0}\left(g \otimes M_{z}^{*} k_{0}\right) \\
& =\left(M_{z}^{*} M_{z}\right)^{-1} M_{z}^{*} M_{z}-0=1
\end{aligned}
$$

and

$$
\begin{aligned}
S g & =S_{0}\left(1-g \otimes k_{0}\right) g=S_{0} g-S_{0}\left(g, k_{0}\right) g \\
& =S_{0} g-g(0) S_{0} g=0 . \quad \square
\end{aligned}
$$

(b) If $S$ is a left inverse of $M_{z}$, then clearly $\operatorname{dim} \operatorname{ker} S=1$. If $g \in \operatorname{ker} S, g \neq 0$, then $g \notin \operatorname{ran} M_{z}$, i.e. $g(0) \neq 0$. Hence there is exactly one $g \in \operatorname{ker} S$ with $g(0)=1$.

Hence there is a 1-1 correspondence between left inverses $S$ of $M_{z}$ and functions $g$ in $\mathscr{H}$ with $g(0)=1$. Furthermore, if $h \in \operatorname{ker} S, h \neq 0$, then $g=h / h(0) \in \operatorname{ker} S$, $g(0)=1$ and $h$ and $g$ have the same zeros including multiplicities. The next proposition tells us about the connection between $\sigma(S)$ and the zeros of $g$.

Proposition 5.2. Suppose $\Omega=\mathbf{D}$ and $\sigma\left(M_{z}\right)=\mathbf{D}^{-}$.

If $g \in \operatorname{ker} S, g \neq 0$, then

$$
\sigma(S)=\mathbf{D}^{-} \cup\{1 / \lambda: \lambda \in Z(g)\} .
$$

Proof. (a) $\mathbf{D}^{-} \cup\{1 / \lambda: \lambda \in Z(g)\} \subseteq \sigma(S)$.

First we shall show that $\mathbf{D} \subseteq \sigma(S)$. We know that $g \in \operatorname{ker} S$, thus $0 \in \sigma(S)$. If $\lambda \in \mathbf{D}$, then $\left(1-\lambda M_{z}\right)$ is invertible, hence $S-\lambda=S-\lambda S M_{z}=S\left(1-\lambda M_{z}\right)$ is not invertible. Thus $\mathbf{D}^{-} \subseteq \sigma(S)$.

Now suppose $\lambda \in \mathbf{D}$ and $g(\lambda)=0$. Since $g(\lambda)=0$, there is a function $f \in \mathscr{H}$ such that $(z-\lambda) f=g$. We have

$$
0=S g=S(z-\lambda) f=(1-\lambda S) f,
$$

hence $1 / \lambda$ is an eigenvalue of $S$.

We have shown that $\mathbf{D}^{-} \cup\{1 / \lambda: \lambda \in Z(g)\} \subseteq \sigma(S)$.

(b) $\sigma(S) \subseteq \mathbf{D}^{-} \cup\{1 / \lambda: \lambda \in Z(g)\}$. 
Suppose $\mu \in \sigma(S)$. If $|\mu| \leqslant 1$, then there is nothing to prove, so we may assume that $\mu=1 / \lambda$ for $|\lambda|<1$. We have to show that $g(\lambda)=0$. We distinguish two cases:

Case $1.1 / \bar{\lambda} \notin \sigma_{\text {ap }}\left(S^{*}\right)$.

In this case $S^{*}-1 / \bar{\lambda}$ is bounded below, hence it has a closed range. $1 / \bar{\lambda} \in \sigma\left(S^{*}\right)$ implies thus that $\operatorname{ran}\left(S^{*}-1 / \bar{\lambda}\right)^{\perp}=\operatorname{ker}(S-1 / \lambda) \neq\{0\}$. It follows that there is an $f \in \mathscr{H}, f \neq 0$ such that

$$
\begin{aligned}
0 & =(1 / \lambda-S) f=(1 / \lambda)\left(S M_{z}-\lambda S\right) f \\
& =(1 / \lambda) S\left(M_{z}-\lambda\right) f .
\end{aligned}
$$

We see that $(z-\lambda) f \in \operatorname{ker} S$, thus there is an $\alpha \in \mathbf{C}$ such that $g=\alpha(z-\lambda) f$. Hence $g(\lambda)=0$.

Case 2. $1 / \bar{\lambda} \in \sigma_{\text {ap }}\left(S^{*}\right)$.

In this case there is a sequence $\left\{f_{n}\right\}_{n \in \mathbf{N}}$ of unit vectors in $\mathscr{H}$ such that $(1 / \bar{\lambda})\left(1-\bar{\lambda} S^{*}\right) f_{n}=\left(1 / \bar{\lambda}-S^{*}\right) f_{n} \rightarrow 0$. We can find complex numbers $\left\{c_{n}\right\}_{n \in \mathbf{N}}$ and vectors $\left\{g_{n}\right\}_{n \in \mathbf{N}} \subseteq \mathscr{H}$ such that $S^{*} f_{n}=c_{n} k_{\lambda}+g_{n}$, where $g_{n} \perp k_{\lambda}$ for all $n \in \mathbf{N}$. With this decomposition we have

$$
\left(M_{z}^{*}-\bar{\lambda}\right) g_{n}=\left(M_{z}^{*}-\bar{\lambda}\right)\left(c_{n} k_{\lambda}+g_{n}\right)=\left(M_{z}^{*}-\bar{\lambda}\right) S^{*} f_{n}=\left(1-\bar{\lambda} S^{*}\right) f_{n} \rightarrow 0 \text {. }
$$

$M_{z}^{*}-\bar{\lambda}$ is bounded below on the orthocomplement of its kernel, thus $g_{n} \rightarrow 0$. It follows that $S^{*} f_{n}-c_{n} k_{\lambda} \rightarrow 0 . S^{*}$ is bounded below, hence $k_{\lambda} \in\left(\operatorname{ran} S^{*}\right)^{-}=$ $(\operatorname{ker} S)^{\perp}$. This means that $g(\lambda)=\left(g, k_{\lambda}\right)=0$, because $g \in \operatorname{ker} S$.

At this point we remark that there is yet another relationship between a left inverse $S$ and $g \in \operatorname{ker} S$, namely that $g$ is a cyclic vector for $\left(M_{z}, \mathscr{H}\right)$ if and only if $S \in \mathscr{B}_{1}(\mathbf{D})$. We shall not prove this here, because we do not see how to use it for our purposes.

5.3. A structure theorem for invariant subspaces with the codimension one property. Proposition 5.2 tells us that finding a function $g \in \mathscr{H}$ which has no zeros in $\mathbf{D}$ is equivalent to finding a left inverse $S$ of $M_{z}$ with $\sigma(S) \subseteq \mathbf{D}^{-}$. We can now prove the following theorem.

TheOREM 5.3. Suppose $\Omega=\mathbf{D}, \sigma\left(M_{z}\right)=\mathbf{D}^{-}$, and $\left\|M_{z} f\right\| \geqslant\|f\|$ for all $f \in \mathscr{H}$.

If $\mathscr{M} \in \operatorname{Lat}\left(M_{z}, \mathscr{H}\right), \operatorname{cod} \mathscr{M}=1$, and $f_{0} \in \mathscr{M} \ominus z \mathscr{M}, f_{0} \neq 0$, then

$$
\begin{gathered}
{\left[f_{0}\right] \subseteq \mathscr{M} \subseteq H^{2} f_{0} \cap \mathscr{H},} \\
\|\varphi\|_{H^{2}}\left\|f_{0}\right\|_{\mathscr{H}} \leqslant\left\|\varphi f_{0}\right\|_{\mathscr{H}} \quad \forall \varphi \in H^{2} \text { such that } \varphi f_{0} \in \mathscr{M} .
\end{gathered}
$$

Proof. (a) First suppose $\mathscr{M}=\mathscr{H}$. Recall that $S_{0}=\left(M_{z}^{*} M_{z}\right)^{-1} M_{z}^{*}$ is a left inverse of $M_{z}$ and $k_{0} \in \operatorname{ker} M_{z}^{*}=\operatorname{ker} S_{0}$.

If $f \in \mathscr{H}$ then there is an $f_{1} \in \mathscr{H}$ such that $f=\alpha k_{0}+z f_{1}$ for some $\alpha \in \mathbf{C}$. We have

$$
\|f\|^{2}=|\alpha|^{2}\left\|k_{0}\right\|^{2}+\left\|z f_{1}\right\|^{2} \geqslant\left\|f_{1}\right\|^{2}
$$

by assumption. Hence

$$
\left\|S_{0} f\right\|=\left\|S_{0}\left(\alpha k_{0}+z f_{1}\right)\right\|=\left\|S_{0} z f_{1}\right\|=\left\|f_{1}\right\| \leqslant\|f\| .
$$

Therefore $S_{0}$ is a contraction and $\sigma\left(S_{0}\right) \subseteq \mathbf{D}^{-}$. It follows from Proposition 5.2 that $k_{0}$ does not have any zeros in $\mathbf{D}$. 
If $f_{0} \in \mathscr{H} \ominus z \mathscr{H}$, then there is an $\alpha \in \mathbf{C}$ such that $k_{0}=\alpha f_{0}$. Hence it is enough to show (5.1) and (5.2) for $k_{0}$.

Now fix $f \in \mathscr{H}$ and set $\varphi=f / k_{0}$. To establish (5.1) we have to show that $\varphi \in H^{2}$. By the above $\varphi$ is an analytic function on $\mathbf{D}$. Thus

$$
\varphi(z)=\sum_{n=0}^{\infty} a_{n} z^{n} \quad \text { and } \quad \varphi \in H^{2} \Leftrightarrow \sum_{n=0}^{\infty}\left|a_{n}\right|^{2}<\infty .
$$

In this case $\|\varphi\|_{H^{2}}^{2}=\sum_{n=0}^{\infty}\left|a_{n}\right|^{2}$.

For $N \in \mathbf{N} \cup\{0\}$ set

$$
g_{N}(z):=\sum_{n=N}^{\infty} a_{n} z^{n-N} k_{0}(z) .
$$

Taking the first summand out of the sum we see that

$$
g_{N}(z)=a_{N} k_{0}(z)+z g_{N+1}(z) .
$$

Claim. For all $N \in \mathbf{N} \cup\{0\}$ we have $g_{N} \in \mathscr{H}$ and

$$
\|f\|_{\mathscr{H}}^{2} \geqslant \sum_{n=0}^{N-1}\left|a_{n}\right|^{2}\left\|k_{0}\right\|_{\mathscr{H}}^{2}+\left\|g_{N}\right\|_{\mathscr{H}}^{2}
$$

Proof. We shall prove the claim by induction on $N$.

If $N=0$, then $g_{0}=f$ and there is nothing to prove.

Suppose now that $N \in \mathbf{N} \cup\{0\}, g_{N} \in \mathscr{H}$ and (5.5) is true. Since $z g_{N+1}=g_{N}-$ $a_{N} k_{0} \in \mathscr{H}$ (see (5.4)) it follows from axiom (1.4) that $g_{N+1} \in \mathscr{H}$. Using the induction hypothesis (5.5) and the fact that $k_{0} \perp z \mathscr{H}$ we get

$$
\begin{aligned}
\|f\|_{\mathscr{H}}^{2} & \geqslant \sum_{n=0}^{N-1}\left|a_{n}\right|^{2}\left\|k_{0}\right\|_{\mathscr{H}}^{2}+\left\|g_{N}\right\|_{\mathscr{H}}^{2} \\
& =\sum_{n=0}^{N-1}\left|a_{n}\right|^{2}\left\|k_{0}\right\|_{\mathscr{H}}^{2}+\left\|a_{N} k_{0}+z g_{N+1}\right\|_{\mathscr{H}}^{2} \\
& =\sum_{n=0}^{N-1}\left|a_{n}\right|^{2}\left\|k_{0}\right\|_{\mathscr{H}}^{2}+\left|a_{N}\right|^{2}\left\|k_{0}\right\|_{\mathscr{H}}^{2}+\left\|z g_{N+1}\right\|_{\mathscr{H}}^{2} \\
& \geqslant \sum_{n=0}^{N}\left|a_{n}\right|^{2}\left\|k_{0}\right\|_{\mathscr{H}}^{2}+\left\|g_{N+1}\right\|_{\mathscr{H}}^{2} .
\end{aligned}
$$

This establishes the claim.

It follows from the claim and (5.3) that $\varphi \in H^{2}$ and

$$
\left\|\varphi k_{0}\right\|_{\mathscr{H}}=\|f\|_{\mathscr{H}} \geqslant\|\varphi\|_{H^{2}}\left\|k_{0}\right\| \quad \text { for all } f=\varphi k_{0} \in \mathscr{H} \text {. }
$$

This establishes (5.1) and (5.2) in the case $\mathscr{M}=\mathscr{H}$.

(b) Now suppose that $\mathscr{M} \neq\{0\}$ is arbitrary with the codimension one property. The following uses the construction given in the proof of Proposition 1.13.

There is an analytic function $\psi$ on $\mathbf{D}$ such that $f / \psi$ is analytic for all $f \in \mathscr{M}$, and for every $\lambda \in \mathbf{D}$, there is a function $g_{\lambda} \in \mathscr{M}$ such that $\left(g_{\lambda} / \psi\right)(\lambda) \neq 0$. We set

$$
\mathscr{K}=\{f / \psi: f \in \mathscr{M}\}, \quad\|f / \psi\|_{\mathscr{K}}=\|f\|_{\mathscr{C}} .
$$


$\left(M_{z}, \mathscr{K}\right)$ is unitarily equivalent to $\left(\left(M_{z} \mid \mathscr{M}\right), \mathscr{M}\right)$, and the unitary equivalence is given by $M_{\psi}: \mathscr{K} \rightarrow \mathscr{M}$ (see Proposition 1.13). Thus $f_{0} \in \mathscr{M} \ominus z \mathscr{M}$ implies $g_{0}=f_{0} / \psi$ $\in \mathscr{K} \ominus{ }_{z} \mathscr{K} .\left(M_{z}, \mathscr{K}\right)$ satisfies the hypothesis of part (a) of this proof, hence $g_{0}$ does not have any zeros in $\mathbf{D}$ and

$$
f / f_{0}=\frac{(f / \psi)}{\left(f_{0} / \psi\right)}=\frac{\left(M_{\psi}\right)^{-1} f}{g_{0}} \in H^{2}
$$

for all $f \in \mathscr{M}$. If we let $\varphi=f / f_{0}$, then it also follows from part (a) of the proof that

$$
\begin{aligned}
\|\varphi\|_{H^{2}}\left\|f_{0}\right\|_{\mathscr{H}} & =\left\|\left(M_{\psi}\right)^{-1}\left(\varphi f_{0}\right) / g_{0}\right\|_{H^{2}}\left\|g_{0}\right\|_{\mathscr{K}} \\
& \leqslant\left\|\left(M_{\psi}\right)^{-1}\left(\varphi f_{0}\right)\right\|_{\mathscr{X}}=\left\|\varphi f_{0}\right\|_{\mathscr{H}} .
\end{aligned}
$$

This concludes the proof of Theorem 5.3.

If $k_{0} \in \mathscr{H}$ does not have any zeros in $\mathbf{D}$, then

$$
\mathscr{K}=\left\{f / k_{0}: f \in \mathscr{H}\right\}, \quad\left\|f / k_{0}\right\|_{\mathscr{K}}=\|f\|_{\mathscr{H}}
$$

is a Hilbert space satisfying (1.1)-(1.4) with $\Omega=$ D. $\left(M_{z}, \mathscr{K}\right)$ is unitarily equivalent to $\left(M_{z}, \mathscr{H}\right)$. Furthermore $\mathscr{K}$ is normalized: $1 \in \mathscr{K}$ and $1 \perp \operatorname{ran} M_{z}$. Under the assumptions of Theorem 5.3 we even have $\mathscr{K} \subseteq H^{2}$.

Theorem 5.3 applies to $\left(M_{z}, D_{\alpha}\right), \alpha \geqslant 0$. In particular, if $\alpha \geqslant 1$, then it applies to every invariant subspace (see [23]).

We do not see how one could use Proposition 5.2 to show that every invariant subspace $\mathscr{M}$ of $\left(M_{z}, D_{\alpha}\right), \alpha<0$, which has the codimension one property, contains a function with no extra zeros. However, for $\alpha \leqslant-1$ one can use results of $\mathrm{C}$. Horowitz to show that such functions indeed exist, see [21] for the details.

\section{BIBLIOGRAPHY}

1. E. Amar, Suites d'interpolation pour les classes de Bergman de la boule et du polydisque de $\mathbf{C}^{n}$, Canad. J. Math. 30 (1978), 711-737.

2. S. Axler, Bergman spaces and their operators, Function Theoretic Operator Theory Conference, Indiana Univ., November 1985.

3. Z_ Zero multipliers of Bergman spaces, Canad. Math. Bull. 28 (1985), 237-242.

4. H. Bercovici, The algebra of multiplication operators on Bergman spaces, Preprint.

5. H. Bercovici, C. Foiaş and C. Pearcy, Dual algebras with applications to invariant subspaces and dilation theory, CBMS Regional Conf. Ser. in Math., no. 56, Amer. Math. Soc., Providence, R. I., 1985.

6. A. Beurling, On two problems concerning linear transformations in Hilbert space, Acta Math. 81 (1949), 239-255.

7. P. Bourdon, Cellular-indecomposable shifts and Beurling's theorem, Michigan Math. J. 33 (1986), $187-193$.

8. L. Brown and B. Korenblum, Cyclic vectors in $A^{-\infty}$, Preprint.

9. L. Brown and A. Shields, Cyclic vectors in the Dirichlet space, Trans. Amer. Math. Soc. 285 (1984), 269-304.

10. S. Brown, B. Chevreau and C. Pearcy, Contractions with rich spectrum have invariant subspaces, J. Operator Theory 1 (1979), 123-136.

11. L. Carleson, On a class of meromorphic functions and its associated exceptional sets, Appelbergs Boktryckeri, Uppsala, 1950.

12. C. Cowen and L. Rubel, $A$ joint spectrum for shift invariant subspaces of $H^{2}$ of the polydisc, Proc. Roy. Irish Acad. 80A (1980), 233-243.

13. M. J. Cowen and R. G. Douglas, Complex geometry and operator theory, Acta Math. 141 (1978), $187-261$. 
14. On moduli for invariant subspaces, Invariant Subspaces and Other Topics, 6th Internat.

Conf. on Operator Theory, Timisoara and Herculane (Romania), June 1-11, 1981, pp. 65--73.

15. H. Grauert, Analytische Faserungen über holomorph vollständigen Räumen, Math. Ann. 135 (1958), 263-273.

16. J. Janas, A note on invariant subspaces under multiplication by $z$ in Bergman space, Proc. Roy. Irish Acad. 83A (1983), 157-164.

17. T. Kato, Perturhation theory for linear operators, 2nd ed., Grundlehren Math. Wiss., Springer-Verlag. New York, 1976.

18. D. Luecking and L. Rubel, Complex analysis. A functional analysis approach, Springer-Verlag, New York, 1985.

19. J. Moeller. On the spectra of some translation incariant spaces, J. Math. Anal. Appl. 4 (1962), $276-296$

20. R. F. Olin and J. E. Thomson, Cellular-indecomposable subnormal operators, Integral Equations and Operator Theory, Vol. 7, 1984, pp. 392-430.

21. S. Richter, On multiplication operators on Banach spaces of analytic functions, Ph.D. dissertation, Univ. of Michigan, 1986.

22. Unitary equivalence of invariant subspaces of Bergman and Dirichlet spaces, Preprint.

23. S. Richter and A. Shields, Bounded analytic functions in the Dirichlet space, Preprint.

24. J. Shapiro, Cyclic inner functions in Bergman spaces, Unpublished Seminar Notes, Univ. of Wisconsin-Madison. July 1980.

25. A. L. Shields, Weighted shift operators and analytic function theory, Topics in Operator Theory (C. Pearcy ed.). Math. Surveys, no. 13, Amer. Math. Soc., Providence, R. I., 1974, pp. 49-128.

26. A. L. Shields and L. J. Wallen, The commutants of certain Hilhert space operators, Indiana Univ. Math. J. 20 (1971), 777-788.

27. G. D. Taylor, Multipliers on $D_{\alpha}$, Trans. Amer. Math. Soc. 123 (1966), 229-240.

28. S. Walsh, Ciclic vectors for the hackward Bergman shift. Dissertation, Univ. of California, Berkeley, 1984.

Department of Mathematics, University of Virginia, Charlottesville, Virginia 22903 